\title{
Purinergic signaling mediates neuroglial interactions to generate sighs
}

Jan Marino Ramirez ( $\nabla$ nino1@uw.edu )

Seattle Children's Research Institute

\section{Liza Severs}

Seattle Children's Research Institute, The University of Wasington

\section{Nicholas Bush}

Seattle Children's Research Institute

\section{Lely Quina}

Seattle Children's Research Institute

\section{Nicholas Burgraff}

Seattle Children's Research Institute

\section{Tatiana Dashevskiy}

Seattle Children's Research Institute

\section{Nathan Baertsch}

Seattle Children's Research Institute https://orcid.org/0000-0003-1589-5575

\section{Biological Sciences - Article}

Keywords: preBötzinger Complex, breathing, sigh, astrocytes, glia, purinergic signaling, neuroglial interactions, rhythm generator, hypoxic response

Posted Date: March 15th, 2021

DOI: https://doi.org/10.21203/rs.3.rs-294601/v1

License: (c) (i) This work is licensed under a Creative Commons Attribution 4.0 International License.

Read Full License 
11 Postal Address for all authors:

$1219009^{\text {th }}$ Ave.

13 JMB 10

14 Seattle, WA 98101

Acknowledgements: This work was supported by the following National Institutes of Health Grants: R01 HL151389, R01 HL126523, and P01 HL090554. We would also like to thank Dr. Marlusa Karlen-Amarante for her overwhelming support overall and for guidance and training for in vivo procedures.

Author Contributions: Conceptualization: J.M.R, T.D., L.J.S., N.A.B.; Methodology:

L.J.S., N.A.B., L.A.Q.; Investigation: L.J.S., N.J.B., N.E.B., N.A.B., L.A.Q., T.D.; Formal Analysis: L.S., N.E.B.; Writing-Original Draft: L.S., N.A.B., T.D., J.M.R.; Writing-Review \& Editing: J.M.R., N.A.B., N.E.B.; Funding Acquisition: J.M.R, L.J.S., T.D.; Visualization: L.S., N.A.B; Supervision: J.M.R

Declaration of Interests: The authors declare no competing interests. 
32 Keywords: preBötzinger Complex, breathing, sigh, astrocytes, glia, purinergic signaling

33 neuroglial interactions, rhythm generator, hypoxic response

34 
Abstract

Sighs prevent the collapse of alveoli in the lungs, initiate arousal under hypoxic conditions, and even express sadness and relief. Sighs are periodically superimposed on normal breaths, known as eupnea. Implicated in the generation of these rhythmic behaviors is the preBötzinger complex (preBötC) ${ }^{1}$. Yet how this small microcircuit can produce two rhythms with strikingly different periodicities remains unresolved. Our computational simulations predict that sighs are generated by the coincidence of two temporally distinct calcium oscillations and are in agreement with experimental evidence suggesting that astrocytes drive sigh behavior through slower, extrinsically driven calcium oscillations that link the eupnea and sigh rhythms. We found that purinergic signaling is necessary to generate spontaneous and hypoxia- induced sighs, and photo-activation of preBötC astrocytes is sufficient to elicit sigh activity. We conclude that sighs are an emergent property of the preBötC network generated by neuroglial interactions, where the distinct modulatory responses of neurons and glia allow for both rhythms to be independently regulated.

Main

A question that is fundamental to the understanding of respiratory physiology is how the activity of a single population of neurons can be reconfigured to produce multiple behaviors, as is observed with eupnea, sighs, and gasps ${ }^{2}$. The sigh is a critical component of breathing, serving to periodically reinflate collapsed alveoli in the lungs. The precise mechanisms by which sighs are generated remain unclear. However, it is understood that sighs and eupnea can be modulated separately ${ }^{3-7}$ and are characterized by distinct intrinsic ${ }^{8}$ and synaptic mechanisms within the preBötc ${ }^{9}$. 
60 Contrary to this idea, it has been suggested that the control of sighing is driven by

61 peptidergic projections from the Retrotrapezoid Nucleus (RTN) to the preBötC ${ }^{7}$.

62 However, lesioning the RTN does not alter sigh frequency ${ }^{10}$, and sighs are preserved in wedges that physically isolate the preBötC ${ }^{5}$. These findings led us to combine

64 computational and experimental approaches to revisit the question of how sighs can be generated locally.

Characterization of sighs: sighs are not generated through inhibitory mechanisms

Sighs vary in shape and not every large amplitude burst is a sigh. Thus, we analyzed characteristics that distinguish sighs from eupneic bursts generated by the preBötC in brainstem slices from neonatal mice. Under control conditions, sigh

71 amplitude, burst duration, and post-burst interval were significantly greater than eupneic

72 bursts, and occurred on a much slower time scale (Fig. 1a, b). Variation in sigh shape is

73 a result of different degrees of connection to the eupneic rhythm; some sighs appeared

74 as connected double bursts, with a large amplitude burst superimposed on a eupneic

75 burst ('biphasic'), whereas others occurred as a single large amplitude burst

76 ('monophasic') (Extended Data Fig. 1a). Biphasic and monophasic sighs did not differ in

77 burst amplitude, burst duration, or post burst interval. Under control conditions in vitro,

78 we found that $78.5 \pm 5.8 \%$ of total sighs were biphasic and $21.4 \pm 5.8 \%$ were

79 monophasic. The prevalence of each sigh shape was variable between slices, and

80 faster eupneic rhythms tended to have more monophasic sighs (Extended Data Fig.

81 1b). Previous hypotheses suggested that sighs are generated through inhibitory

82 mechanisms ${ }^{11}$. We show that pharmacological blockade of GABAergic and glycinergic

83 synaptic inhibition with strychnine and gabazine, referred to as "inhibition block", did not

84 eliminate sighs, change their frequency, or dissociate them from eupneic bursts (Fig. 
1c) $)^{12}$. Sigh amplitude, post-burst interval, duration, and shape were also unaffected by inhibition block. However, the amplitude of eupneic bursts increased relative to sighs ${ }^{13}$,

87 thereby decreasing the ratio of sigh to eupnea amplitude (Extended Data Fig. 1c). Moreover, sigh frequency could still be increased pharmacologically following inhibition block with the $\beta$-adrenergic agonist isoproterenol (Fig. 1c), a potent sigh modulator ${ }^{6}$.

\section{An excitatory preBötC model combines $\mathrm{Ca}^{2+}$ oscillations to generate sighs}

Based on these results, we designed an in silico model of the preBötC network comprised of 300 excitatory model neurons. To test the feasibility of calcium oscillations to generate sigh-like network activity, the in silico excitatory preBötC network modifies an existing model ${ }^{14}$ to include a current gated by intracellular calcium $\left(\mathrm{Ca}^{2+}{ }_{i n}\right.$; Extended data Fig. 1d). $\mathrm{Ca}^{2+}$ in evolves on both fast and slow timescales (Fig. 1d-f, Table 1, 2). The fast calcium signal $\left(\mathrm{Ca}^{2+}\right.$ fast $)$ is linked to inactivation of persistent sodium channels (Eq. 17) and activates during periods of burst firing (i.e. eupnea) ${ }^{15}$. The slow calcium $\left(\mathrm{Ca}^{2+}{ }^{+}\right.$slow $)$follows a slow oscillatory pattern ${ }^{15,16}$ that is extrinsically driven and synchronized across all neurons. This slow $\mathrm{Ca}^{2+}$ oscillation occurs independently of neuronal activity in the network and follows previously described dynamics ${ }^{16}$. Thus, the total $\mathrm{Ca}^{2+}{ }_{\text {in }}$ of each neuron of the respiratory network is a superposition of calcium 103 dynamics on these two timescales (Eq.17).

105 based on each neuron's firing pattern in synaptic isolation (Fig. 1d, Extended Data Fig.

106 3). Simulations including $\mathrm{Ca}^{2+}$ gated channels did not produce sigh bursts in the

107 absence of $\mathrm{Ca}^{2+}$ oscillations. Neither the addition of a fast, eupnea-linked $\mathrm{Ca}^{2+}$

108 oscillation, nor a slow, extrinsically driven $\mathrm{Ca}^{2+}$ oscillation was sufficient to produce sigh 109 bursts on their own. However, model networks including both fast and slow oscillations 
110 reliably produced periodic sigh-like bursts similar to those observed in vitro ${ }^{5}$ (Fig. $1 \mathrm{~d}, \mathrm{e}$ ).

111 Indeed, compared to eupneic bursts, sigh bursts in the computational model were larger

112 in amplitude and exhibited prolonged burst durations and post-burst intervals (Extended

113 Data Fig. 1e). Modelled sighs were also temporally linked to the eupneic rhythm often

114 occurring as an augmented population burst superimposed on a normal eupneic burst.

115 Consistent with experimental findings in vitro, inclusion of both slow and fast $\mathrm{Ca}^{2+}$

116 components produced sigh-like bursts in "bursting" neurons even when synaptically

117 isolated from the network (Fig. 1d) ${ }^{5}$. Furthermore, the model produced a small

118 subpopulation of "sigh-only" neurons $(18 / 300,6 \%)$ that were active during sighs but not

119 eupnea (Fig. 1e, f) $)^{5}$. These neurons were not defined to be "sigh-only" a priori.

120 To further test the role of $\mathrm{Ca}^{2+}$ oscillations in producing the sigh rhythm, $\mathrm{Ca}^{2+}$

121 dynamics were manipulated in the model network. We altered the amplitude of the slow

$122 \mathrm{Ca}^{2+}$ oscillation by modifying the parameter $k 1$, the amplitude of the fast $\mathrm{Ca}^{2+}$ oscillation

123 by modifying $k 2$, and the frequency of the slow $\mathrm{Ca}^{2+}$ oscillation by modifying the

124 intracellular calcium release parameter $[\mathrm{IP} 3]^{16}$. Increasing the amplitude of $\mathrm{Ca}^{2+}$ slow

125 increased the probability of sighs (Fig. $2 \mathrm{a}, \mathrm{b}$ ). When the amplitude of $\mathrm{Ca}^{2+}$ slow was small,

126 total $\mathrm{Ca}^{2+}$ exceeded the $\mathrm{Ca}^{2+}$ gated channels' threshold and only evoked a sigh when

127 the $\mathrm{Ca}^{2+}$ slow burst coincided with the eupnea linked $\mathrm{Ca}^{2+}{ }_{\text {fast }}$ oscillation, thereby linking the

128 timing of sigh bursts to the burst phase of the eupnea rhythm (Fig. 2c). Indeed, sighs

129 rarely occurred when the $\mathrm{Ca}^{2+}$ slow peak occurred early in eupneic phase (see Methods),

130 that is, when the $\mathrm{Ca}^{2+}$ slow transient immediately followed a eupneic burst (Fig. 2c,

131 Extended Data Fig. 2a) ${ }^{16,17}$. Increasing $\mathrm{Ca}^{2+}$ slow amplitude increased the probability of a

132 given $\mathrm{Ca}^{2+}$ slow oscillation evoking a sigh by lengthening the time-window within the

133 eupneic cycle during which the total $\mathrm{Ca}^{2+}{ }_{\text {in }}$ could exceed the threshold for $\mathrm{Ca}^{2+}$ gated

134 channel activity (Extended Data Fig. 2a). The morphology of modeled sighs also 
135 depended on the amplitude and eupneic phase of the $\mathrm{Ca}^{2+}$ slow oscillation (Extended

136 Data Fig. 2b, d). Biphasic sighs occurred more often at low $\mathrm{Ca}^{2+}$ slow amplitudes and

137 when the $\mathrm{Ca}^{2+}$ slow oscillation coincided with or slightly preceded the onset of a eupneic

138 burst. Conversely, monophasic sighs occurred more frequently when $\mathrm{Ca}^{2+}$ slow amplitude

139 was high, and when the $\mathrm{Ca}^{2+}$ slow oscillation peaked between eupneic bursts. While

140 holding the dynamics of the $\mathrm{Ca}^{2+}$ slow 0 scillation fixed, sighs also emerged from the model

141 preBötC network when the amplitude of $\mathrm{Ca}^{2+}{ }_{\text {fast }}$ was increased; however, sighs were

142 only generated within a small range of $\mathrm{Ca}^{2+}{ }_{\text {fast }}$ amplitudes (Extended Data Fig. 3).

143 Increasing the frequency of $\mathrm{Ca}^{2+}$ slow while holding the $\mathrm{Ca}^{2+}$ slow amplitude constant led to

144 a corresponding increased sigh frequency from low to moderate $\mathrm{Ca}^{2+}$ slow frequencies

145 (Extended Data Fig. 3). At higher $\mathrm{Ca}^{2+}{ }_{\text {slow }}$ frequencies sighs were eliminated, and

146 eupnea was disrupted (Fig. 2d).

\section{$\mathrm{Ca}^{2+}$ dynamics drive sigh behavior in silico and in vitro}

Our in silico preBötC model network made three predictions for the role of an

150 extrinsically driven calcium oscillation in generating sigh bursts. Increases in $\left[\mathrm{Ca}^{2+}\right]_{\text {in }}$

151 should: 1) increase sigh occurrence, 2) decrease the prevalence of biphasic sigh bursts,

152 and 3) abolish sighs above a certain critical concentration. To test these predictions

153 experimentally, we used norepinephrine (NE), a neuromodulator known to increase sigh

154 frequency ${ }^{6,18}$ and up-regulate $\mathrm{Ca}^{2+}{ }_{\text {in }}$ concentration ${ }^{19}$ through second messenger

155 pathways via activation of adrenergic receptors ${ }^{17}$. NE increased the frequency of sighs

156 in a manner consistent with the simulated manipulation of $\mathrm{Ca}^{2+}$ slow frequency (Fig. 2d,

157 e). Furthermore, increasing [NE] in vitro and increasing slow $\mathrm{Ca}^{2+}$ oscillation amplitude

158 in silico had similar effects on sigh shape, shifting sighs from mostly biphasic to mostly

159 monophasic (Fig. 2e, f). Consistent with simulated population activity observed at high 
$160 \mathrm{Ca}^{2+}$ slow frequencies (Fig. 2d), inter-sigh interval decreased with increasing [NE] until the

161 concentration was too high and resulted in cessation of sighs, leaving only eupneic

162 activity (Fig. 2e, g). Collectively, these experimental and computational manipulations of

$163 \mathrm{Ca}^{2+}$ suggest sigh generation is driven by the presence of a slow, rhythmic signaling

164 mechanism that causes synchronized increases in $\left[\mathrm{Ca}^{2+}{ }_{\text {in }}\right.$ among preBötC neurons.

166 Astrocytes modulate sighing through purinergic signaling

We hypothesized that glia could be the source of the $\mathrm{Ca}^{2+}$ slow transients that are synchronized across the respiratory rhythm generating network. Astrocytes respond to metabolic changes in their extracellular environment by increasing intracellular $\mathrm{Ca}^{2+20}$

170 through G-protein coupled receptor cascades. In the respiratory networks ${ }^{21,22}$, and

171 throughout the central nervous system ${ }^{23,24}$, astrocyte responses spread through gap

172 junctions ${ }^{25}$ and involve the release of ATP which modulates neural activity via purinergic

173 mechanisms ${ }^{26-28}$. Therefore, we tested the contribution of purinergic signaling to sigh

174 generation in vitro by pharmacological manipulation of ADP and ATP receptors P2Y1

175 and P2X, respectively (Fig. 3a, b) ${ }^{29}$. In rhythmic brainstem slices containing the

176 preBötC, activation of purinergic $\mathrm{P} 2 \mathrm{Y} 1$ receptors $\left(\mathrm{P} 2 \mathrm{Y} 1_{\mathrm{R}}\right)$ with MRS2365 significantly

177 increased sigh frequency (Fig. 3a), and subsequent blockade of P2Y1 receptors

178 (MRS2279), but not $\mathrm{P} 2 \mathrm{X}$ receptors (TNP-ATP), abolished sighs. The $\mathrm{P} 2 \mathrm{Y} 1_{\mathrm{R}}$ antagonist

179 also blocked sighs induced with the $\beta$-adrenergic agonist isoproterenol (Fig. 3b). These

180 findings suggest that $\mathrm{P} 2 \mathrm{Y} 1$ - but not $\mathrm{P} 2 \mathrm{X}$-dependent purinergic signaling is important for 181 the generation of spontaneous sighs.

182 To test if purinergic P2Y1 inhibition would also block sighs generated during the 183 central hypoxic response in vitro (Fig. 3d), eupnea and sigh activity was recorded before 184 and during an initial bout of severe hypoxia (SH). After a recovery period, MRS2279 
was applied prior to a second hypoxic episode. Inhibition of P2Y1 signaling eliminated

or reduced sighs in 8 of 9 slices. We performed immunohistochemistry to confirm P2Y $1_{R}$ expression in the brainstem ${ }^{29,30}$. We found that $P 2 Y 1_{R}$ was widely expressed in the preBötC and appeared to colocalize with both neurons and astrocytes (Fig. 3c).

189 Furthermore, inhibition of $\mathrm{P} 2 \mathrm{Y} 1_{\mathrm{R}}$ significantly reduced the augmentation of eupneic 190 frequency characteristic of the initial phase of the hypoxic response ${ }^{26}$. In control

191 experiments where no drugs were applied, similar effects were not observed during the second exposure to severe hypoxia (Fig. 3d).

Astrocytes are sufficient for sigh generation within the preBötC network

To further test the potential role of astrocytes in sigh generation, we tested

196 whether selective manipulation of astrocytes ${ }^{31,32}$ would be sufficient to produce sighs. In

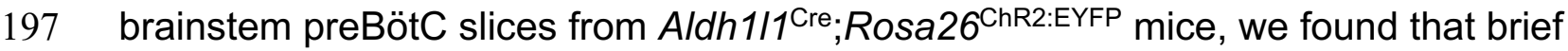

198 (200ms) light pulses evoked both eupnea and sigh bursts recorded from the contralateral preBötC (Fig. 4a,b). Our photostimulation parameters were able to reliably evoke bursts in 12/25 slice preparations. In the remaining slices, longer duration or

201 higher intensity light pulses were effective in evoking eupnea and sigh bursts. Sighs

202 evoked by photoactivation of Aldh1/1-expressing cells were indistinct from spontaneous

203 sighs in burst amplitude and post burst interval (Fig. 4a). However, the duration of 204 evoked sigh bursts was shorter relative to spontaneous sighs, consistent with a small 205 increase in the proportion evoked monophasic sighs. These experiments suggest that 206 Aldh1/1 astrocytes are sufficient to initiate both sigh and eupneic bursts that are largely 207 indistinguishable from spontaneously occurring activities.

208 Blocking $P / Q$-type calcium channels inhibits sighs ${ }^{9}$, and $P / Q$ type (Cav2.1) 209 knockout mice do not generate sighs in vitro or in vivo, and ultimately die of atelectasis ${ }^{8}$. 
210 The effect of P/Q-type channel can also be mimicked by the general $\mathrm{Ca}^{2+}$ channel

211 blocker $\mathrm{Cd}^{2+}$ which selectively inhibits sighs at low concentrations ${ }^{5,33}$. Therefore, $4 \mu \mathrm{M}$

$212 \mathrm{Cd}^{2+}$ was applied during optogenetic stimulation to test if astrocyte stimulation would

213 continue to evoke sighs (Fig. 4b, Extended Data Fig. 4a). In $\mathrm{Cd}^{2+}$, photostimulation

214 failed to evoke sighs. However, we observed evoked double bursts and bursts that

215 appeared to have slightly larger amplitudes than eupneic bursts. We refer to these

216 bursts as 'sigh attempts'. Sigh attempts were less likely to occur than sighs evoked

217 under baseline conditions; $4.7 \%$ of stimulations evoked a sigh attempt in $\mathrm{Cd}^{2+}$,

218 compared to $24.1 \%$ in baseline. The amplitude of sigh attempts in $\mathrm{Cd}^{2+}$ was significantly

219 reduced from baseline sighs but remained slightly larger than evoked eupnea. The

220 duration of sigh attempts in $\mathrm{Cd}^{2+}$ was statistically indistinct from eupnea duration.

221 Moreover, sigh attempts in $\mathrm{Cd}^{2+}$ lacked an extended post burst interval ("post-sigh

222 apnea") that is characteristic of normal sighs.

223 We also tested if the P2Y1 $\mathrm{R}$ antagonist MRS2279 would affect the ability of

224 astrocytes to drive sighing (Fig. 4b, Extended Data Fig. 4a). Following P2Y1R inhibition,

225 sighs evoked during photostimulation of Aldh1/1 astrocytes were completely blocked in

226 all but one slice $(n=7)$. Consistent with evoked sigh attempts observed in $\mathrm{Cd}^{2+}$, sigh

227 attempts in MRS2279 were smaller in amplitude than baseline sighs and had burst

228 durations similar to evoked eupnea. However, post-burst intervals after sigh attempts in

229 MRS2279 remained longer than those after eupnea (Fig. 4b). Collectively, these data

230 indicate that $P 2 Y 1_{R}-$ and $\mathrm{Ca}^{2+-}$ dependent signaling are important mechanisms that

231 allow astrocytes to drive sighing activity in the preBötC.

232 Next, we asked if light activation of astrocytes in vivo would have the same effect

233 on sigh modulation $(n=9)$. Indeed, we found that there was a higher probability of

234 evoking sighs in vivo than in vitro (Fig. 4c, Extended Data Fig. 4c), and in general, sigh 
235 characteristics similarly matched in vitro data. Evoked sighs in vivo could be

236 unambiguously distinguished from eupnea by characterizing the diaphragm amplitude

237 (Fig. 4d). Notably, evoked eupnea in simultaneous recordings of the hypoglossal nerve

238 (XII) tended to be larger in amplitude than spontaneous eupnea, which made

239 distinguishing sighs from eupnea more difficult based solely on XII recordings

240 (Extended Data Fig. 4b).

241 Interestingly, MRS2279 bilaterally injected into the preBötC in vivo was less

242 effective in completely blocking spontaneous or evoked sighs $(n=3 / 6$, Extended Data

243 Fig. 4c, d); contrary to the very reliable inhibitory effect observed in vitro (Fig. 4b). This

244 may not be surprising given that numerous descending inputs that are present in vivo

245 and endogenously modulate sighs ${ }^{34}$ including bombesin-like peptides ${ }^{7}$ and

246 noradrenergic inputs ${ }^{6}$ will likely reduce the ability to block sighs by purinergic

247 mechanisms in vivo. Corresponding to this variability, we observed a higher probability

248 of evoked burst failures altogether in the presence of MRS2279 (Extended Data Fig.

249 4c). This weaker stimulation corroborates the control of sighs from outside areas ${ }^{7}$, which

250 are eliminated from in vitro slice experiments. Our data indicate that sighs are an

251 emergent property involving local neuroglial interactions mediated by purinergic

252 signaling and modulatory inputs intrinsic and extrinsic to the preBötC.

254 Materials and Methods

255 Computational Model

256 The computational model for the present study incorporates a shared external

257 source of $\mathrm{Ca}^{2+}$ oscillations to elicit global synchronization across the respiratory

258 network. Equations were adopted from an original model of the respiratory network ${ }^{14}$.

259 The membrane potential of each compartment and gating variables are described in 
260 equations 5 - 16. A Ca ${ }^{2+}$ dependent current which has bell shaped $\mathrm{Ca}^{2+}$

261 activation/inactivation was incorporated into each neuron. Based on the previous

262 experimental observations indicating that the sigh frequency is independent from

263 eupneic frequency ${ }^{2}$ and that sighs are inhibited by $\mathrm{Ca}^{2+}$ channel blockers and absent in

$264 \mathrm{P} / \mathrm{Q}$ type $\mathrm{Ca}^{2+}$ channel knock-out mice ${ }^{8}$, we modeled the dynamics of $\mathrm{Ca}^{2+}$ oscillations

265 as a superposition of two independent components: 1) slow oscillations with dynamics

266 governed by $\mathrm{IP}_{3}$ channels in endoplasmic reticulum $(\mathrm{ER})^{16,35}$ and 2 ) fast $\mathrm{Ca}^{2+}$ that are

267 associated with eupneic activity and are regulated by the dynamics of the persistent

268 sodium current ${ }^{4,35}$. The slow component is defined by:

$$
\begin{gathered}
\frac{d C}{d t}=f_{i} \cdot\left(J_{E R_{\text {in }}}-J_{E R_{\text {out }}}\right) \\
J_{E R_{\text {in }}}=\left(L+P\left(\frac{I \cdot C \cdot l}{\left(I+k_{I}\right)\left(C+k_{a}\right)}\right)^{3}\right)\left(\frac{C_{t}-C}{\sigma}-C\right) \\
J_{E R_{\text {out }}}=\left(\frac{V_{e} C^{2}}{k_{e}^{2}+C^{2}}\right) \\
l^{\prime}=A \cdot\left(k_{d}-l \cdot\left(C+k_{d}\right)\right)
\end{gathered}
$$

269 where $C$ is the shared external calcium signal, $J_{E R_{\text {in }}}$ and $J_{E R_{o u t}}$ are the fluxes of $\mathrm{Ca}^{2+}$ into 270 and out of the ER, respectively, and $l$ is the $\mathrm{IP}_{3}$ channel gating variable. The parameter

$271 f_{i}$ is the ratio of bound to free $\left[\mathrm{Ca}^{2+}\right], L$ is the leak ER permeability, $P$ is the maximum

272 permeability of the $\mathrm{IP}_{3}$ channels, $I$ is the concentration of $\mathrm{IP}_{3}$ channels, $k_{I}$ is the

273 dissociation constant of $\mathrm{IP}_{3}$ receptor by $\mathrm{IP}_{3}, k_{a}$ is the dissociation constant of the $\mathrm{IP}_{3}$

274 receptor for $\mathrm{Ca}^{2+}, C_{t}$ is the maximal $\left[\mathrm{Ca}^{2+}\right]$ in the external source, $\sigma$ is the ratio of the

275 volume of the ER to the volume of the cytosol, $V_{e}$ is the maximal SERCA pump rate, $k_{e}$

276 is the SERCA pump coefficient, $\mathrm{A}$ is a scaling factor, and $k_{d}$ is the constant of $\mathrm{IP}_{3}$

277 receptor inactivation by $\mathrm{Ca}^{2+}$.

278 Each neuron is a single compartment neuron modeled by the following equations: 


$$
\frac{d V}{d t}=\frac{-1}{C_{m}}\left(I_{N a}+I_{K}+I_{N a P}+I_{l e a k}+I_{s y n}+I_{C a s}\right)
$$

279 The sodium, potassium, persistent sodium, leak, synaptic, and Calcium sensitive

280 currents are, respectively:

$$
\begin{array}{cc}
I_{N a}=g_{N a} \cdot m_{\infty}(V)^{3} \cdot(1-n(V)) \cdot\left(V-E_{N a}\right) & \text { Eq.6 } \\
I_{K}=g_{K} \cdot n(V)^{4} \cdot\left(V-E_{K}\right) & \text { Eq.7 } \\
I_{N a P}=g_{N a P} \cdot m_{p_{\infty}}(V) \cdot h(V) \cdot\left(V-E_{N a}\right) & \text { Eq.8 } \\
I_{\text {leak }}=g_{\text {leak }} \cdot\left(V-E_{\text {leak }}\right) & \text { Eq.9 } \\
I_{\text {syn }}=\sum_{i}^{k} g_{\text {syn }} \cdot\left(V-E_{\text {syn }}\right) & \text { Eq. } 10 \\
I_{\text {Cas }}=g_{\text {Cas }} \cdot m_{\text {Cas }}(C a)^{2} \cdot h_{\text {Cas }}(C a) \cdot\left(V-E_{\text {Cas }}\right) & \text { Eq.11 }
\end{array}
$$

281 where $g_{x}$ represents the maximal conductance of each current, $E_{x}$ represents the

282 reversal potential of the respective channel.

283 The dynamic activation $\left(n, m_{\text {Cas }}\right)$ and inactivation $\left(h, h_{\text {Cas }}\right)$ of the currents are governed 284 by:

$$
\frac{d x}{d t}=\frac{x_{\infty}-x}{\tau_{x}}
$$

286 for $x=\left\{n, h, m_{\text {Cas }} h_{\text {Cas }}\right\}$. Time constants $\tau_{x}$ are

$$
\tau_{x}=\frac{\overline{\tau_{x}}}{\cosh \left(\frac{V-V_{x}}{2 \sigma_{x}}\right)} \text { for: } x=\{n, h\}
$$

288 and

$$
\tau_{x}=\tau_{0_{x}}+\frac{\overline{\tau_{x}}}{1+\exp \left(\xi_{x}\left([C a]_{i n}-[C a]_{x}\right)\right)} \text { for: } x=\left\{m_{\text {Cas }}, h_{\text {Cas }}\right\}
$$

289 Steady state voltage dependent activation or inactivation, $x_{\infty}$ is defined as:

290 and

$$
x_{\infty}=\frac{1}{1+\exp \left(\frac{V-V_{x}}{\sigma_{x}}\right)} \text { for: } x=\left\{m, m_{p}, h, n\right\}
$$




$$
x_{\infty}=\frac{1}{1+\exp \left(\theta_{x}\left([C a]_{i n}-[C a]_{x}\right)\right)} \text { for: } x=\left\{m_{\text {Cas }}, h_{\text {Cas }}\right\}
$$

292 The values of $[\mathrm{Ca}]_{m_{C a s}}$ and $[\mathrm{Ca}]_{h_{C a s}}$ define the opening and closing (respectively) of the

$293 \mathrm{Ca}^{2+}$ "activation window" (the range of $[\mathrm{Ca}]_{i n}$ values that open the $\mathrm{Ca}^{2+}$ sensitive

294 channel), the values of $\xi_{m_{C a s}}, \xi_{h_{C a s}}$ define the kinetics of the $\mathrm{Ca}^{2+}$ sensitive channel

295 activation and inactivation (respectively), and the values of $\theta_{m_{C a s}} \theta_{h_{C a s}}$ define the

296 steepness of the sigmoidal activation/inactivation of the $\mathrm{Ca}^{2+}$ sensitive channel.

297 Intracellular calcium concentration dynamics are described as:

$$
\frac{d[C a]_{\text {in }}}{d t}=\frac{1}{\tau_{C a}}\left(k_{1} \cdot C+\left(k_{2} \cdot m_{p_{\infty}} \cdot h\right)-[C a]_{\text {in }}\right)
$$

298 Where the first term describes the slow external calcium dynamics and the second term 299 describes the fast, bursting driven internal dynamics. The variables $l, C$ are those from

300 the slow, external calcium oscillation. $k_{1}, k_{2}$ govern the relative influence of the slow and

301 fast calcium sources, respectively, on the total intracellular calcium levels: $[\mathrm{Ca}]_{\text {in }}$.

302 Synapses from a source neuron $(i)$ to a target neuron $(j)$ were governed by the 303 equations ${ }^{36}$ :

$$
\begin{gathered}
\frac{d s_{i}}{d t}=\frac{\left(1-s_{i}\right) m_{\infty_{s y n}}\left(V_{i}\right)-s_{i}}{\tau_{\text {syn }}} \\
m_{\infty_{\text {syn }}}=\frac{1}{1+\exp \left(\frac{V_{i}-Q_{s}}{\sigma_{s y n}}\right)} \\
g_{\text {syn }_{i}}=g_{\text {syn } \max } \cdot s_{i}
\end{gathered}
$$

305 Networks were constructed of 300 neurons with randomly generated excitatory synaptic

306 connections such that the average neuron had a total of 6 synaptic connections

307 (incoming and outgoing inclusive). Eight unique network configurations (connectivity

308 patterns) were generated to allow for multiple replicates in some experiments. Neurons 
309 were defined such that their activity without synaptic inputs could be clearly defined as

310 bursting, tonic, or quiescent by varying their leak conductance $g_{\text {leak }}$ (Fig. 2a).

311 Parameters that are shared across all neurons are given in Table 1. Network and cell

312 specific parameters are given in Table 2. Simulations were constructed using the Brian2

313 python package ${ }^{37}$ using the Runge-Kutta $4^{\text {th }}$ order method with a fixed timestep of

$3140.25 \mathrm{~ms}$. Networks were simulated for $1000 \mathrm{~s}$, unless otherwise stated. After $1000 \mathrm{~s}$,

315 synaptic conductance $\left(g_{s y n}\right)$ was set to $0 n S$ to observe isolated neuron behavior. Spike

316 times were computed as the time at which the cell voltage crossed a $-20 \mathrm{mV}$ threshold.

317 Population rate was computed by first taking the sum of the number of spikes in each

318 timestep, then smoothing with a boxcar filter with width of $10-100 \mathrm{~ms}$. Eupneic phase

319 was defined as the time difference between the peak of the $\mathrm{Ca}^{2+}$ slow signal and the peak

320 of the previous eupneic burst, divided by the mean eupneic interval.

322 Animals

323 All experiments and animal procedures were approved by the Seattle Children's

324 Research Institute's Animal Care and Use Committee and conducted in accordance

325 with the National Institutes of Health guidelines. Male and female mice were selected

326 randomly. Experiments were performed in brainstem slices obtained from CD1 or

327 transgenic mice age postnatal day 4-12 and bred at Seattle Children's Research

328 Institute. Aldh1/1 cre mice were obtained from Jackson Laboratories (FVB-Tg (Aldh1l1-

329 cre) JD1884Htz/J, Jax stock No. 023748). Cre mice were crossed with homozygous

330 mice containing a floxed STOP channelrhodopsin2 fused to an EYFP (Ai32) reporter

331 sequence. All mice were group housed with access to food and water ad libitum in a

332 temperature controlled $\left(22 \pm 1^{\circ} \mathrm{C}\right)$ facility with a $12 \mathrm{hr}$ light/dark cycle. 
In vitro transverse brainstem slice preparation and population recordings

The following techniques used are described in detail in a series of publications

336 by the Ramirez laboratory ${ }^{38,4,9,9}$. PreBötC slice thickness varied between $590-610 \mu \mathrm{M}$.

337 Animals were quickly decapitated, and brainstems were rapidly dissected in cold

338 artificial cerebrospinal fluid (ACSF). Serial transverse slices were made from the

339 appearance facial nerve until reaching the level of the preBötC, where a 580-610uM

340 slice was made. Slices were transferred into the recording chamber with circulating

341 ACSF with osmolarity of $305-320$ osm/L, $\mathrm{pH} 7.4$ and containing (in $\mathrm{mM}$ ) $\mathrm{NaCl}(118), \mathrm{KCl}$

342 (3), $\mathrm{CaCl}_{2}$ (1.5), $\mathrm{MgCl}_{2}$ (1), $\mathrm{NaHCO}_{3}$ (25), $\mathrm{NaH}_{2} \mathrm{PO}_{4}$ (1), and D-glucose (30).

343 Temperature was maintained at $29-31^{\circ} \mathrm{C}$ and ACSF was bubbled with carbogen $(95 \%$

$344 \mathrm{O}_{2} / 5 \% \mathrm{CO}_{2}$ ). During extracellular population recordings the superfused ACSF was

345 circulating and recycled at a flow rate of $10-13 \mathrm{ml} / \mathrm{min}$. Activity was recorded from the

346 caudal side of the transverse slice. Some experiments were performed with two slices

347 at the same time using two extracellular electrodes. Extracellular raw traces were

348 filtered and integrated. The data obtained were analyzed using GraphPad Prism 7 and

349 PCLAMP 10 software which is available online at

350 http://www.moleculardevices.com/systems/conventional-patch-clamp/pclamp-10-

351 software. For unknown reasons, some preBötC slices do not have periodic sighs within

352 the baseline recording period, or they occur at intervals $>20$ mins. These slices were not

353 used for experiments. PreBötC slices were not used across multiple drug experiments.

354 When selecting bursts for doublet analysis, bursts occurring in quick succession were

355 only characterized as doublets if the first burst did not reach baseline before onset of

356 the second burst. 
In vivo anesthetized experiments were performed as previously published ${ }^{39}$.

360 Briefly, adult mice were anesthetized with urethane $(1.5 \mathrm{mg} / \mathrm{kg}$, i.p.) and placed supine 361 on a custom surgical table. Adequate depth of anesthesia was determined via heart 362 rate, breathing frequency, and response to toe pinch. Bipolar stainless steel electrodes

363 were implanted in the diaphragm muscles to perform electromyographic recordings of 364 inspiratory motor activities ${ }^{40}$. The trachea was exposed through a midline incision and 365 cannulated caudal to the larynx with a curved (180 degree) tracheal tube (24 G). The 366 trachea and esophagus were removed rostral to the tracheotomy, and underlying 367 muscles were removed to expose the basal surface of the occipital bone. Mice were 368 supplied with $100 \% \mathrm{O}_{2}$ throughout the remainder of the surgery and experimental 369 protocol. The portion of the occipital bone and dura overlying the ventral medullary 370 surface were removed and the brainstem surface was perfused with aCSF $\left(36{ }^{\circ} \mathrm{C}\right)$ 371 equilibrated with carbogen $(95 \%$ O2, 5\% CO2). The hypoglossal nerve (XII) was then 372 isolated unilaterally and recorded from using a fire-polished pulled glass pipette filled 373 with aCSF. XII electrical activity was amplified (10,000x), filtered (low pass $300 \mathrm{~Hz}$, high 374 pass $5 \mathrm{kHz}$ ), rectified, integrated, and digitized (Digidata $1550 \mathrm{~A}$, Axon Instruments). In 375 animals in which we observed baseline sighs, MRS2279 (500 $\mu \mathrm{M}, 41.3 \mathrm{~nL})$ and Evan's 376 Blue dye or red fluorospheres were co-injected bilaterally in 3 positions around the 377 preBötC using a Nanolnject, as to avoid lesioning the preBötC core and altering the 378 rhythm. In most instances, injections close to preBötC stimulated sighs immediately 379 following placement of the cannula. Saline injected control animals did not have any 380 alterations in baseline breathing, and we observed the same increase in sighs in most 381 injections $(n=4 / 5)$. Similar to in vitro experiments, many animals in vivo did not have 382 baseline sighs and therefore MRS2279 was not injected. 
A glass fiber optic $(200 \mu \mathrm{m}$ core, $0.24 \mathrm{NA})$ connected to a blue $(447 \mathrm{~nm})$ highpowered LED was positioned above the preBötC contralateral to the extracellular

387 electrode or XII nerve and positioned for maximal response. Power was set to 0.5

$388 \mathrm{~mW} / \mathrm{mm}^{2}$. Fifty continuous 20 s sweeps were recorded for each stimulation experiment

389 in vitro, and 20 sweeps for in vivo experiments. Laser power settings and pulse width

390 were selected based on previous experiments ${ }^{13,41}$. In approximately $1 / 3$ of

391 Aldh1/1 cre/Ai32 preBötC slices, we had difficulty evoking any responses unless

392 increasing the wavelength to a higher laser power $\left(>0.5 \mathrm{~mW} / \mathrm{mm}^{2-1} \mathrm{~mW} / \mathrm{mm}^{2}\right)$. Despite

393 having robust respiratory rhythms, we did not continue to perform stimulations in these

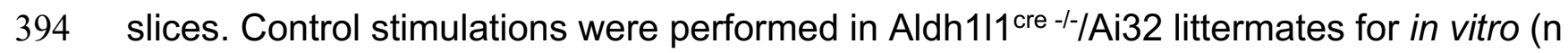

$395=2)$ and adults for in vivo $(\mathrm{n}=3)$ experiments. There were no observed non-specific

396 effects of light stimulation, even at maximum laser power.

$397 \quad$ Norepinephrine (1-40 $\mu \mathrm{M}, \mathrm{DL}-$ Norepinephrine hydrochloride, cat. A7256),

398 Isoproterenol (Isoprenaline hydrochloride, cat. I5627), Strychnine (1 $\mu \mathrm{M}$, cat. S0532),

399 and cadmium (4 $\mu \mathrm{M}$ cadmium chloride, cat. 20208) were purchased from Sigma.

400 Gabazine (1 $\mu \mathrm{M}, \mathrm{SR} 99531$ hydrobromide, cat. 1262), MRS2279 $\left(20 \mu \mathrm{M}, \mathrm{P} 2 \mathrm{Y}_{\mathrm{R}}\right.$

401 antagonist, cat. 2158,), MRS2365 (20 $\mu \mathrm{M}, \mathrm{P} 2 \mathrm{Y} 1_{\mathrm{R}}$ agonist, cat. 2157) and TNP-ATP

402 triethylammonium salt $(20 \mu \mathrm{M}, \mathrm{P} 2 \mathrm{X}$ receptor antagonist, cat. 2464) were obtained from

403 Tocris. All drugs were diluted in water.

405 Histology

After experiments, animals were fixed by transcardial perfusion with cold ACSF

407 followed by 4\% PFA in phosphate buffer solution (PBS). Medullary and cortical tissue 
was carefully extracted and equilibrated in graded sucrose solutions to a final

409 concentration of $30 \%$, embedded in OCT solution, frozen at $-80 \mathrm{C}$, and cryosectioned

410 at $25 \mu \mathrm{m}$ for imaging of immunofluorescence. Slides containing brainstem tissue slices

411 were washed 3x10 min in PBS. Tissue slices were then permeabilized and blocked with

$4121 \%$ normal donkey serum in $0.5 \%$ Triton X-100/PBS for $1 \mathrm{~h}$. Following blocking, tissue

413 slices were incubated with the primary antibody for either NeuN (Millipore MAB377),

414 P2Y1 (Abcam ab140859), or EGFP (Abcam ab6673) (1:500 in 1\% normal donkey

415 serum in $0.5 \%$ Triton X-100/PBS) overnight. After primary antibody incubation, tissue

416 was washed $3 \times 10$ min. in phosphate buffer solution (PBS), followed by secondary

417 antibody incubation for 30 min. (Alexa Fluor 488, 594, or 647). Lastly, tissue was

418 washed 3×10 min. in PBS and hard set mounted (VECTASHIELD hardset mounting

419 media with DAPI). Immunofluorescence images were acquired using an Olympus

420 VS120 Virtual Slide Microscope Scanner. The excitation/emission filter sets used for

421 imaging were DAPI: 380-405/410-480, FITC: 460-490/500-550, Cy3/TRITC: 540-

422 570/580-640, and Cy5: 625-645/655-705. Laser scanning confocal imaging was

423 performed using a Zeiss 710 34-channel Quasar LSC Microscope.

Sigh classification

Custom unsupervised methods were developed to distinguish between eupneic bursts and "sigh attempts" evoked by channelrhodopsin stimulation in in vitro

428 recordings. First, bursts in the integrated pre-Bötzinger recording were detected such

429 that the prominence of each peak was greater than 2.5 times the standard deviation of

430 the integrated activity. The onset of the burst was defined as the time at which the trace

431 reached $20 \%$ of the subsequent peak height. Bursts that were aberrantly large

432 amplitude ( >75 times the mean amplitude) or very fast rising (reached peak amplitude 
433 in less than $150 \mathrm{~ms}$ ) were discarded as noise. The burst shape for all bursts was

434 extracted from the onset to $1.5 \mathrm{~s}$ after onset of the burst. Burst shapes were then

435 decomposed into principal components. The first $n$ components (where $n$ is the number

436 of components needed to explain $95 \%$ of the variance) of the spontaneous bursts (i.e.,

437 not evoked by optogenetic stimulation) were used as features to fit a robust covariance

438 estimator (sci-kit learn MinCovDet). Since the spontaneous bursts were observed never

439 to be putative sighs, this covariance represents the prior distribution of eupnea, that is,

440 the expected eupneic shape. We calculate the Mahalnobis distance $(D)$ of each burst to

441 the mean eupneic shape. $D$ quantifies how similar each burst is to the "average"

442 eupnea. We then determine a threshold as:

$$
D_{\text {thresh }}=D_{\text {spontaneous }}+4 * \operatorname{IQR}\left(D_{\text {spontaneous }}\right)
$$

444 All evoked bursts where $D_{\text {evoked }}>D_{\text {thresh }}$ were considered outliers. This outlier

445 detection included many false positive bursts in which the burst shape was different

446 from the average eupnea, but upon inspection was not typical of a sigh (often long slow

447 bursts or large amplitude noise). We boost this outlier detection with an identical method

448 using instead the intuitive features of burst amplitude, full width at half max (FWHM) and

449 time to next burst (post-burst apnea) as features to the covariance estimator. A burst

450 was considered a sigh attempt when it was determined to be an outlier in both the

451 classifier fit on burst shape and on the intuitive features. We imposed a set of final

452 criteria where a sigh attempt must be larger in amplitude than $90 \%$ of the spontaneous

453 bursts. To classify sighs in in vivo recordings, diaphragm EMG was recorded, rectified,

454 and integrated as described above. Bursts in the integrated diaphragm EMG were

455 detected, and each burst shape was extracted. The area under the curve was computed

456 for each burst. Bursts were labeled as "sighs" if both the area under the curve and the 
457 amplitude for a given sigh was $>6 x$ the median absolute deviation (MAD) of the 20

458 bursts preceding and following the given burst.

Statistical analysis

Analyses were performed using GraphPad Prism5 software. Groups were compared using the appropriate t-tests, one-way or two-way ANOVAs followed by

463 Bonferonni's post hoc tests, if necessary. Statistical significance was set at $\alpha=0.05$.

464 Masking was not used during data collection or analysis.

Code availability

Source code is available at (https://github.com/nbush257/sighnet).

Data availability

The data presented in this manuscript is available upon request.

Caveats and limitations

The use of transverse preBötC slices as a model for respiratory rhythm

474 generation is associated with significant caveats of which oxygenation ${ }^{42}$, incomplete 475 network, low temperature, elevated $[\mathrm{K}+]$ saline are the most obvious concerns, and 476 have been discussed in most of our publications ${ }^{2,4,42-44}$. Although transverse slices

477 generate rhythmicity in $3 \mathrm{mM} \mathrm{K}^{+45-47}$ rhythmicity is more reliable and consistently 478 generated at $8 \mathrm{mM} \mathrm{K}^{+}$. Thus, in the present study we consistently use the extracellular 479 concentration of $8 \mathrm{mM} \mathrm{K}^{+}$. To distinguish the fast-respiratory rhythm from the slow sigh 480 rhythm, we refer to the fast-respiratory rhythm as "eupneic" activity throughout this 481 study. However, we do not imply that this in vitro activity represents "eupnea" in the 
482 behavioral sense. Rather this activity characterizes primarily the inspiratory component

483 of the respiratory activity generated within the preBötC $2,48,49$. Furthermore, sighs were

484 characterized based on their appearance in the in vivo preparation and were carefully

485 distinguished using a novel pipeline aimed at reducing bias. However, in vivo recordings

486 should involve increased diaphragm activity along with the characteristics observed of

487 sighs, and thus in vitro work is limited in this sense. In our analysis, we did not consider

488 doublets to be sighs. Despite these caveats and limitations, the comparisons between

489 the experiments performed in vitro revealed many similarities to the testable predictions

490 set forth by the computational model. 


\section{References}

4921 Smith, J. C., Ellenberger, H. H., Ballanyi, K., Richter, D., W. \& Feldman, J. L.

493 Pre-Bötzinger Complex: A Brainstem Region That May Generate Respiratory

$494 \quad$ Rhythm in Mammals. Science 254, 726-729 (1991).

4952 Lieske, S., Thoby-Brisson, M., Telgkamp, P. \& Ramirez, J. Reconfiguration of the

496

497

4983 Peña, F. \& Ramirez, J.-M. Endogenous activation of serotonin-2A receptors is required for respiratory rhythm generation in vitro. The Journal of Neuroscience 22, 11055-11064 (2002).

5014 Peña, F., Parkis, M. A., Tryba, A. K. \& Ramirez, J. M. Differential contribution of

502

503

504

505 neural network controlling multiple breathing patterns: eupnea, sighs and gasps. Nat Neurosci 3, 600-607 (2000).

506 pacemaker properties to the generation of respiratory rhythms during normoxia and hypoxia. Neuron 43, 105-117 (2004).

508

Tryba, A. K. et al. Differential modulation of neural network and pacemaker activity underlying eupnea and sigh-breathing activities. J Neurophysiol 99, 21142125 (2008).

5107 Li, P. et al. The peptidergic control circuit for sighing. Nature 530, 293-297, 511 doi:10.1038/nature16964 (2016).

5128 Koch, H. et al. Stable respiratory activity requires both P/Q-type and N-type 513 voltage-gated calcium channels. J Neurosci 33, 3633-3645 (2013).

5149 Lieske, S. \& Ramirez, J.-M. Pattern-specific synaptic mechanisms in a multifunctional network. II. Intrinsic modulation by metabotropic glutamate receptors. J Neurophysiol 95, 1334-1344 (2006).

Souza, G. et al. Breathing regulation and blood gas homeostasis after near complete lesions of the retrotrapezoid nucleus in adult rats. J Physiol 596, 25212545, doi:10.1113/JP275866 (2018). of sigh rhythmogenesis in the embryonic mouse. J Physiol 592, 2169-2181, doi:10.1113/jphysiol.2013.268730 (2014). (2020). 
13 Baertsch, N. A., Severs, L. J., Anderson, T. M. \& Ramirez, J. M. A spatially dynamic network underlies the generation of inspiratory behaviors. Proc Natl Acad Sci U S A 116, 7493-7502, doi:10.1073/pnas.1900523116 (2019).

14 Butera, R. J., Jr., Rinzel, J. \& Smith, J. C. Models of respiratory rhythm generation in the pre-Botzinger complex. I. Bursting pacemaker neurons. $J$ Neurophysiol 82, 382-397 (1999).

15 Toporikova, N., Chevalier, M. \& Thoby-Brisson, M. Sigh and Eupnea Rhythmogenesis Involve Distinct Interconnected Subpopulations: A Combined Computational and Experimental Study $(1,2,3)$. eNeuro 2, doi:10.1523/ENEURO.0074-14.2015 (2015).

16 Li, Y. X. \& Rinzel, J. Equations for InsP3 receptor-mediated [Ca2+]i oscillations derived from a detailed kinetic model: a Hodgkin-Huxley like formalism. $J$ Theor Biol 166, 461-473, doi:10.1006/jtbi.1994.1041 (1994).

17 Galaz-Montoya, M., Wright, S. J., Rodriguez, G. J., Lichtarge, O. \& Wensel, T. G. beta2-Adrenergic receptor activation mobilizes intracellular calcium via a noncanonical cAMP-independent signaling pathway. The Journal of biological chemistry 292, 9967-9974, doi:10.1074/jbc.M117.787119 (2017).

18 Viemari, J.-C. \& Ramirez, J.-M. Norepinheprine differentially modulates different types of respiratory pacemaker and nonpacemaker neurons. J Neurophysiol 95, 2070-2082 (2006).

19 Saez, J. C., Moreno, A. P. \& Spray, D. C. Norepinephrine induces Ca2+ release from intracellular stores in rat pinealocytes. $J$ Pineal Res 16, 57-64, doi:10.1111/j.1600-079x.1994.tb00083.x (1994).

20 Angelova, P. R. et al. Functional Oxygen Sensitivity of Astrocytes. J Neurosci 35, 10460-10473, doi:10.1523/JNEUROSCI.0045-15.2015 (2015).

21 Funk, G. D. Neuromodulation: purinergic signaling in respiratory control. Comprehensive Physiology 3, 331-363, doi:10.1002/cphy.c120004 (2013).

22 Ramirez, J. M., Severs, L., Ramirez, S. C. \& Agosto-Marlin, I. M. Advances in cellular and integrative control of oxygen homeostasis within the central nervous system. J Physiol, doi:10.1113/JP275890 (2018).

23 Rodrigues, R. J., Marques, J. M. \& Cunha, R. A. Purinergic signalling and brain development. Semin Cell Dev Biol 95, 34-41, doi:10.1016/j.semcdb.2018.12.001 (2019).

24 Del Puerto, A., Wandosell, F. \& Garrido, J. J. Neuronal and glial purinergic receptors functions in neuron development and brain disease. Front Cell Neurosci 7, 197, doi:10.3389/fncel.2013.00197 (2013). 
56325 Cotrina, M. L. et al. Connexins regulate calcium signaling by controlling ATP release. Proc Natl Acad Sci U S A 95, 15735-15740,

56626 Rajani, V. et al. Release of ATP by preBotzinger complex astrocytes contributes to the hypoxic ventilatory response via a Ca2+ -dependent P2Y1 receptor

27 Chen, L. et al. K(ATP) channels of parafacial respiratory group (pFRG) neurons

28 Rajani, V., Zhang, Y., Revill, A. \& Funk, G. D. The role of P2Y1 receptor

29 Lorier, A. R. et al. P2Y1 Receptor Modulation of the Pre-Bötzinger Complex

30 Huxtable, A. G. et al. Glia contribute to the purinergic modulation of inspiratory Inspiratory Rhythm Generating Network In Vitro. The Journal of Neuroscience 27, 993-1005 (2007). rhythm-generating networks. J Neurosci 30, 3947-3958, doi:10.1523/JNEUROSCI.6027-09.2010 (2010).

Tien, A. C. et al. Regulated temporal-spatial astrocyte precursor cell proliferation involves BRAF signalling in mammalian spinal cord. Development 139, 24772487, doi:10.1242/dev.077214 (2012).

34 Doi, A. \& Ramirez, J. M. Neuromodulation and the orchestration of the respiratory rhythm. Respiratory physiology \& neurobiology 164, 96-104, doi:10.1016/j.resp.2008.06.007 S1569-9048(08)00166-3 [pii] (2008).

35 Thoby-Brisson, M. \& Ramirez, J. M. Identification of two types of inspiratory pacemaker neurons in the isolated respiratory neural network of mice. $J$ Neurophysiol 86, 104-112 (2001).

36 Destexhe, A. Oscillations, complex spatiotemporal behavior, and information transport in networks of excitatory and inhibitory neurons. Phys Rev E Stat Phys 
601

603

604

605

606

607

608

609

610

611

612

613

614

615

616

617

618

619

620

621

622

623

624

625

626

627

628

629

630

631

632

633

634

635

636

637

638

Plasmas Fluids Relat Interdiscip Topics 50, 1594-1606, doi:10.1103/physreve.50.1594 (1994).

37 Stimberg, M., Brette, R. \& Goodman, D. F. Brian 2, an intuitive and efficient neural simulator. Elife 8, doi:10.7554/eLife.47314 (2019).

38 Ramirez, J. M. \& Richter, D. W. The neuronal mechanisms of respiratory rhythm generation. Current opinion in neurobiology 6, 817-825 (1996).

39 Baertsch, N. A., Baertsch, H. C. \& Ramirez, J. M. The interdependence of excitation and inhibition for the control of dynamic breathing rhythms. Nat Commun 9, 843, doi:10.1038/s41467-018-03223-x (2018).

40 Lemes, E. V., Colombari, E. \& Zoccal, D. B. Generation of active expiration by serotoninergic mechanisms of the ventral medulla of rats. J Appl Physiol (1985) 121, 1135-1144, doi:10.1152/japplphysiol.00470.2016 (2016).

41 Baertsch, N. A. \& Ramirez, J. M. Insights into the dynamic control of breathing revealed through cell-type-specific responses to substance P. Elife 8, doi:10.7554/eLife.51350 (2019).

42 Hill, A. A., Garcia, A. J. r., Zenella, S. \& Upadhyaya, R. Graded Reductions in Oxygenation Evoke Graded Reconfiguration of the Isolated Respiratory Network. J Neurophysiol 105, 625-639 (2011).

43 Garcia, A. J., 3rd, Khan, S. A., Kumar, G. K., Prabhakar, N. R. \& Ramirez, J. M. Hydrogen peroxide differentially affects activity in the pre-Botzinger complex and hippocampus. J Neurophysiol 106, 3045-3055 (2011).

$44 \quad$ Zanella, S. et al. When norepinephrine becomes a driver of breathing irregularities: how intermittent hypoxia fundamentally alters the modulatory response of the respiratory network. J Neurosci 34, 36-50, doi:10.1523/JNEUROSCI.3644-12.2014 34/1/36 [pii] (2014).

45 Ruangkittisakul, A. et al. Generation of eupnea and sighs by a spatiochemically organized inspiratory network. J Neurosci 28, 2447-2458, doi:10.1523/JNEUROSCI.1926-07.2008 (2008).

46 Ruangkittisakul, A., Kottick, A., Picardo, M. C., Ballanyi, K. \& Del Negro, C. A. Identification of the pre-Botzinger complex inspiratory center in calibrated "sandwich" slices from newborn mice with fluorescent Dbx1 interneurons. Physiol Rep 2, doi:10.14814/phy2.12111 (2014).

47 Tryba, A. K., Pena, F. \& Ramirez, J. M. Stabilization of bursting in respiratory pacemaker neurons. J Neurosci 23, 3538-3546, doi:23/8/3538 [pii] (2003).

48 Carroll, M. S. \& Ramirez, J. M. Cycle-by-cycle assembly of respiratory network activity is dynamic and stochastic. $J$ Neurophysiol 109, 296-305, doi:10.1152/jn.00830.2011 
49 Harris, K. D. et al. Different roles for inhibition in the rhythm-generating (2017).

643 
a
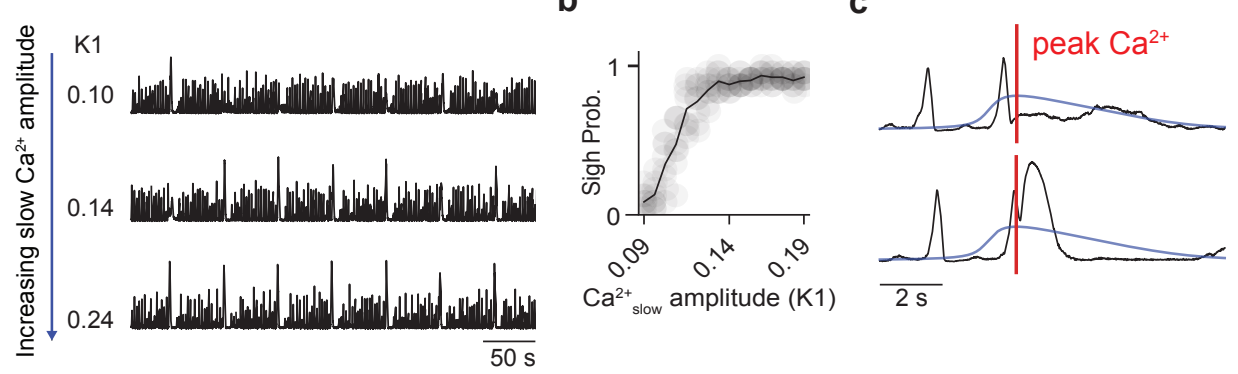

$$
\text { e }
$$

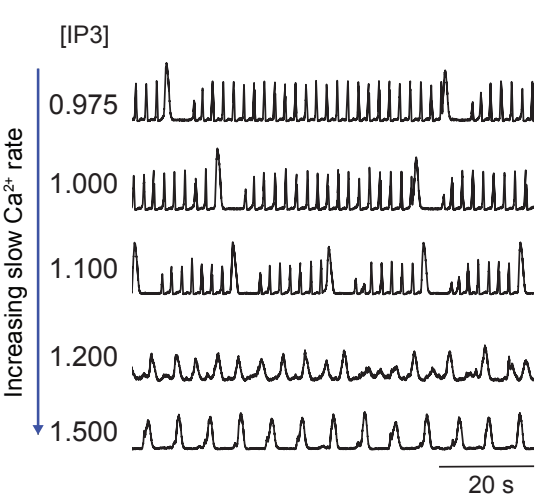



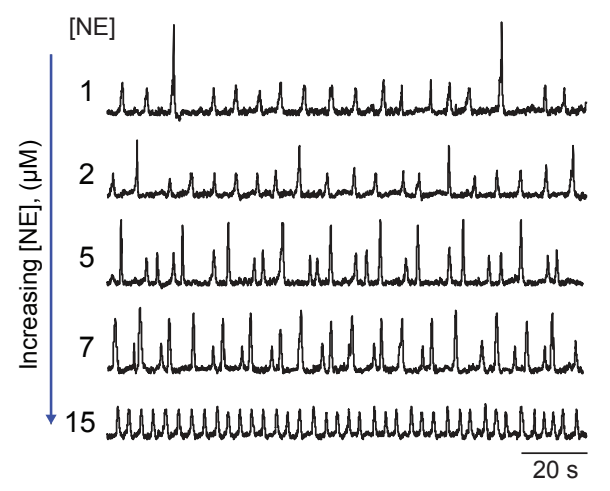

f

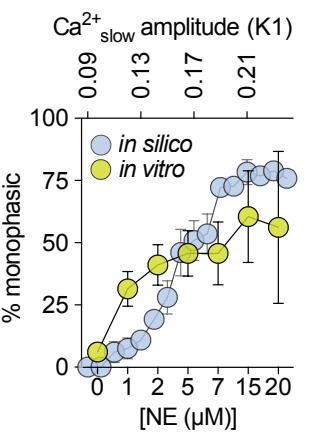

g



Figure 2. Sigh frequency and shape is similarly affected by manipulations of $\mathrm{Ca}^{2+}$ in vitro and in silico. (a) In silico population activity from simulations with increasing amplitude of $\mathrm{Ca}^{2+}{ }_{\text {slow }}$ signals (k1). (b) Sigh probability during an extrinsic $\mathrm{Ca}^{2+}$ burst as a function of slow $\mathrm{Ca}^{2+}$ amplitude (k1) represented by independent simulations (gray circles) and average of 8 simulations (black line). (c) Population traces (black), extrinsic $\mathrm{Ca}^{2+}$ (blue), and peak extrinsic $\mathrm{Ca}^{2+}$ (red) for sigh failure and success. (d) Population firing rates of simulated networks with increasing frequency of extrinsic slow $\mathrm{Ca}^{2+}$ transients as governed by increases in [IP3]. (e) Representative traces of in vitro preBötC population activity; increasing [NE] increases number of sigh bursts. (f) Sighs are more likely to be monophasic as slow $\mathrm{Ca}^{2+}$ amplitude (blue) or [NE] (green) increases. (g) Average inter-sigh interval decreases for in silico simulations (blue) as function of extrinsic $\mathrm{Ca}^{2+}$ oscillation frequency ([IP3]) with fixed amplitude and in vitro recordings as a function of [NE] (green). 




- in vitro
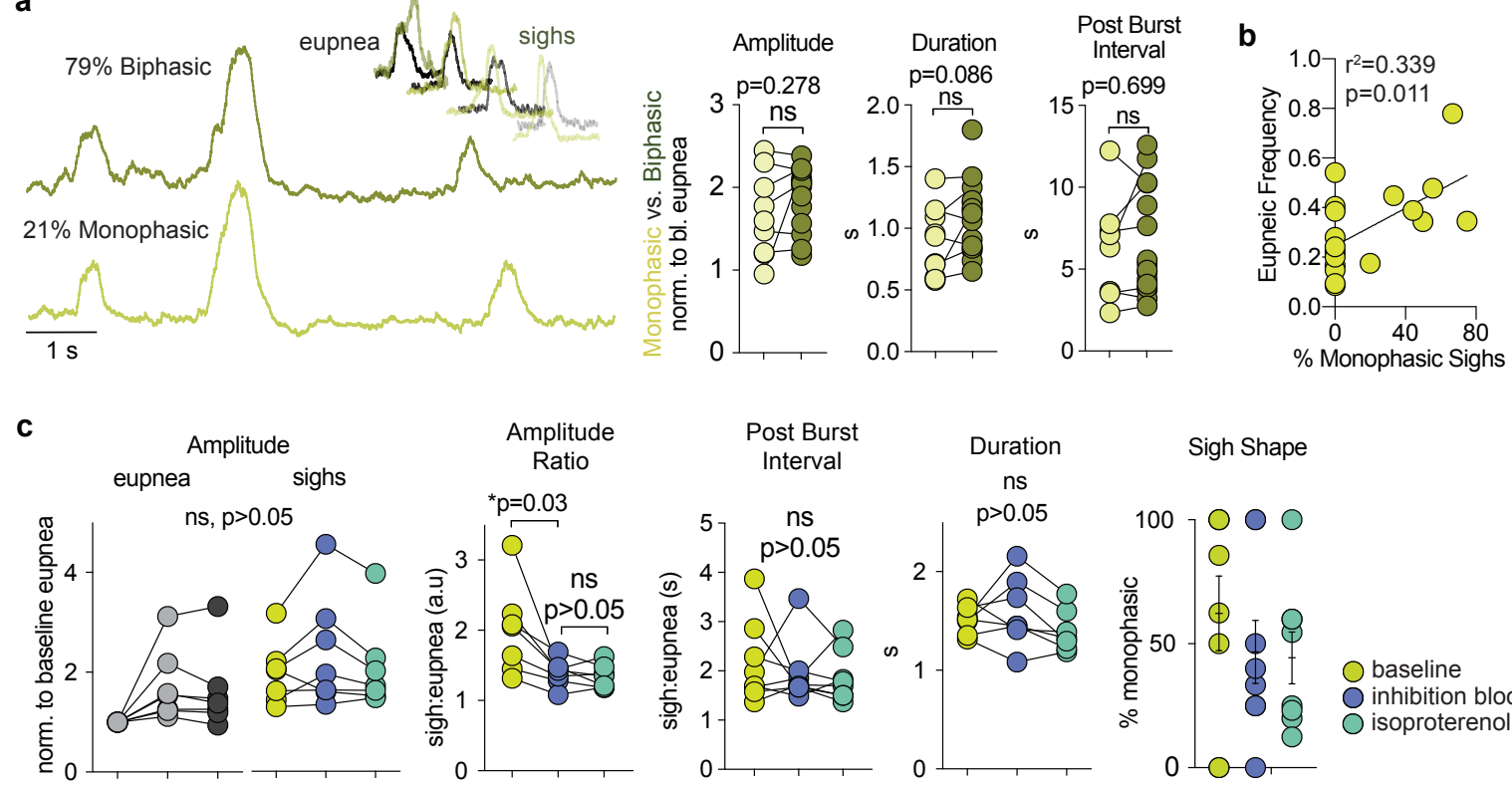

baseline

inhibition block

in silico

d

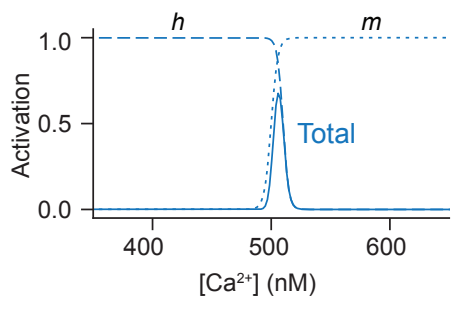

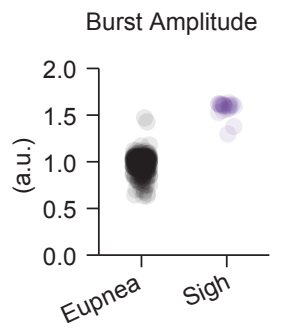
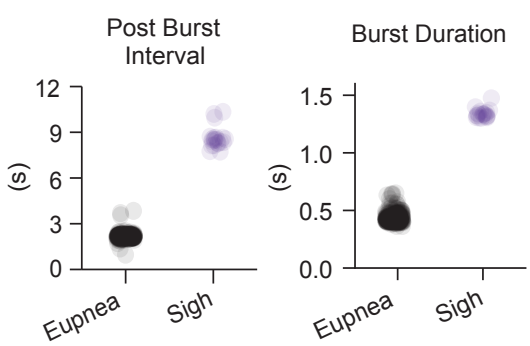

Extended Data Figure 1. (a) Sighs observed in the transverse preBötC slice preparation are commonly 'biphasic' or 'monophasic' but retain the same distinguishing sigh characteristics (paired t-tests). Inset shows overlayed eupnea (gray) and sighs (green) for comparison. (b) Higher eupneic frequencies were positively correlated with a larger number of monophasic sighs. (c) Inhibition block does not alter sigh characteristics or frequency. No change in the amplitude of sighs was observed with inhibition block, however a significant increase in eupneic amplitude led to a decrease in the sigh:eupnea ratio. No change was observed in the sigh burst duration, sigh post burst interval, or sigh shape (ordinary one-way ANOVA). (d) $\mathrm{Ca}^{2+}$-sensitive cation channel steady state activation curves. Steady state activation $\mathrm{m}_{\text {Cas } \infty}$, inactivation $\left(\mathrm{h}_{\text {Cas } \infty}\right)$, and total activation $\left(\mathrm{m}^{2}{ }_{\text {Cas } \infty} \cdot \mathrm{h}_{\text {Cas } \infty}\right)$ of the $\mathrm{Ca}^{2+}$-sensitive channels are shown as a function of intracellular $\mathrm{Ca}^{2+}$ concentration. Activation starts at zero for low [Ca]in; inactivation starts at 1 for low $\left[\mathrm{Ca}^{2+}\right]_{\text {in }}$. These channels open only when the $\mathrm{Ca}^{2+}{ }_{\text {in }}$ levels fall in the center "activation window". The curves follow the expressions in equation 16; the onset and offset of the "activation window" are governed by the parameters $[\mathrm{Ca}]_{\text {mcas }}$ and $[\mathrm{Ca}]_{\text {hcas' }}$, respectively. (e) in silico sigh burst amplitude, post-burst interval, and burst durations are larger than eupneic bursts, consistent with in vitro results (see Fig. 1a). Each dot represents one burst. 


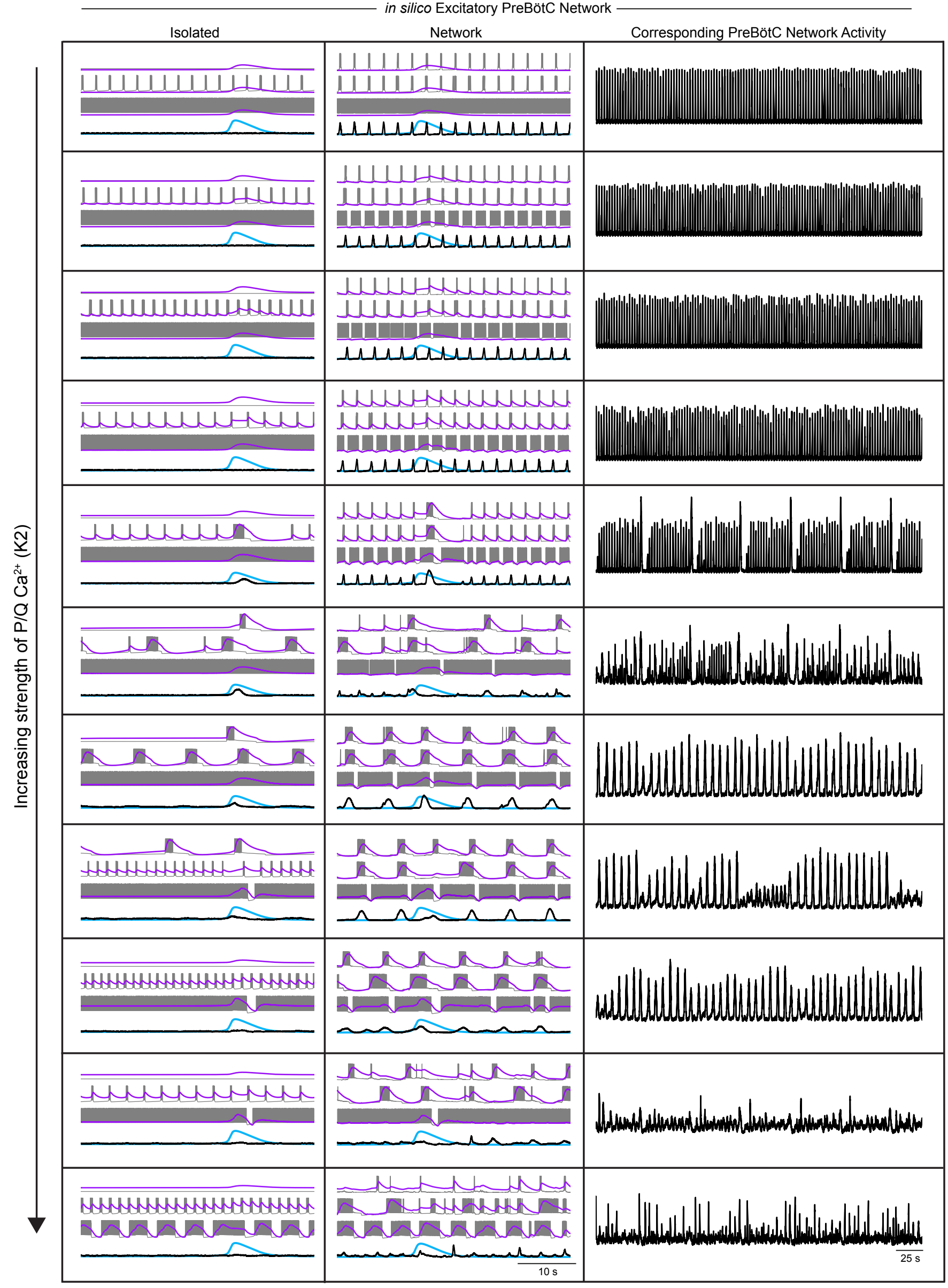

Extended Data Figure 3. Modulating $\mathrm{P} / \mathrm{Q}$-type $\mathrm{Ca}^{2+}$ strength modulates network and sigh bursting behaviors. Example single neuron and population traces from networks while varying the strength of the $P / Q$ type $\mathrm{Ca}^{2+}$ current (k2). Each row shows voltage (gray) and [Ca] $]_{\mathrm{i}}$ (magenta) traces for 3 example neurons (quiescent, bursting, and tonic, top to bottom); the integrated population spiking activity (black); and the slow extrinsically generated $\mathrm{Ca}^{2+}$ signal (blue). Far left columns show the synaptically isolated neurons, middle column shows the synaptically connected network, and far right column shows the integrated population activity for the connected network over an expanded time frame. 

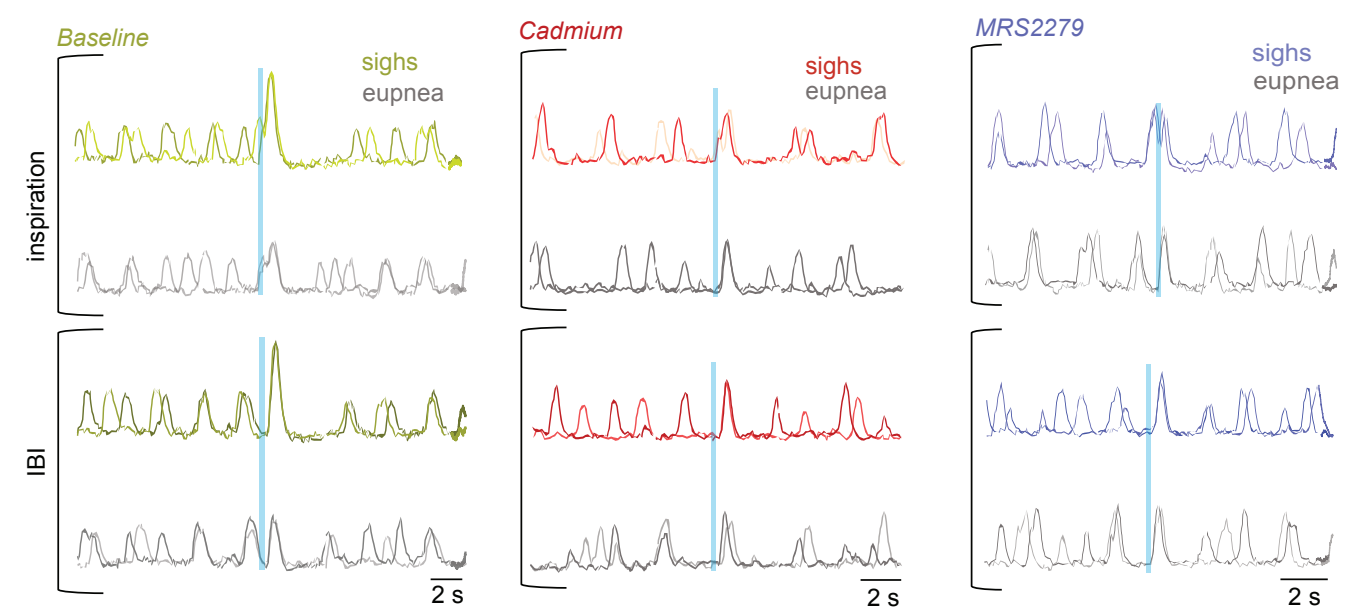

b

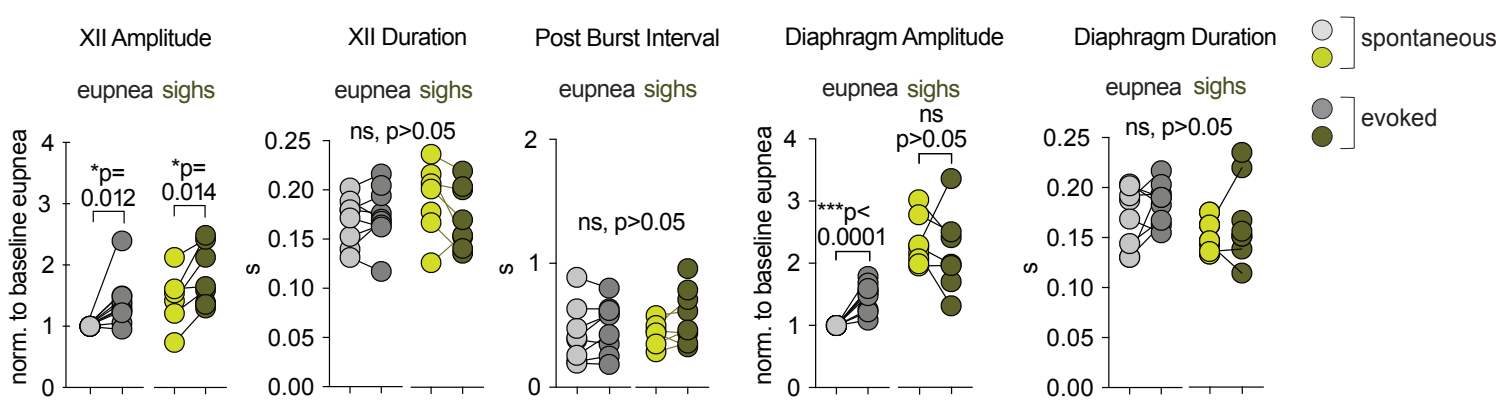

c

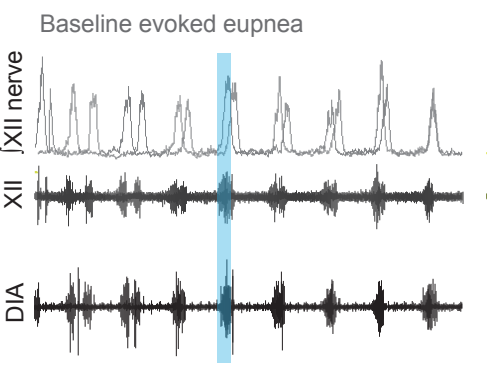

Baseline evoked sighs

MRS2279 evoked eupnea

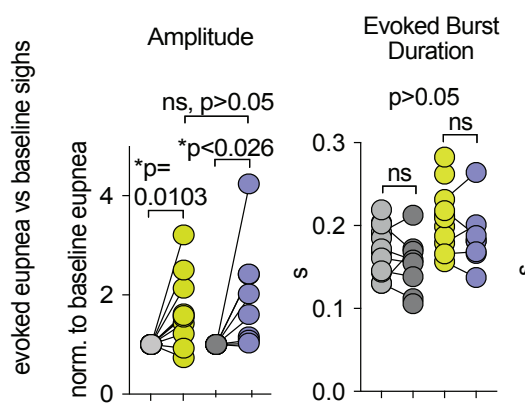

Evoked Post Burst
Interval $\begin{gathered}\text { Evoked Diaphragm } \\ \text { Amplitude }\end{gathered}$
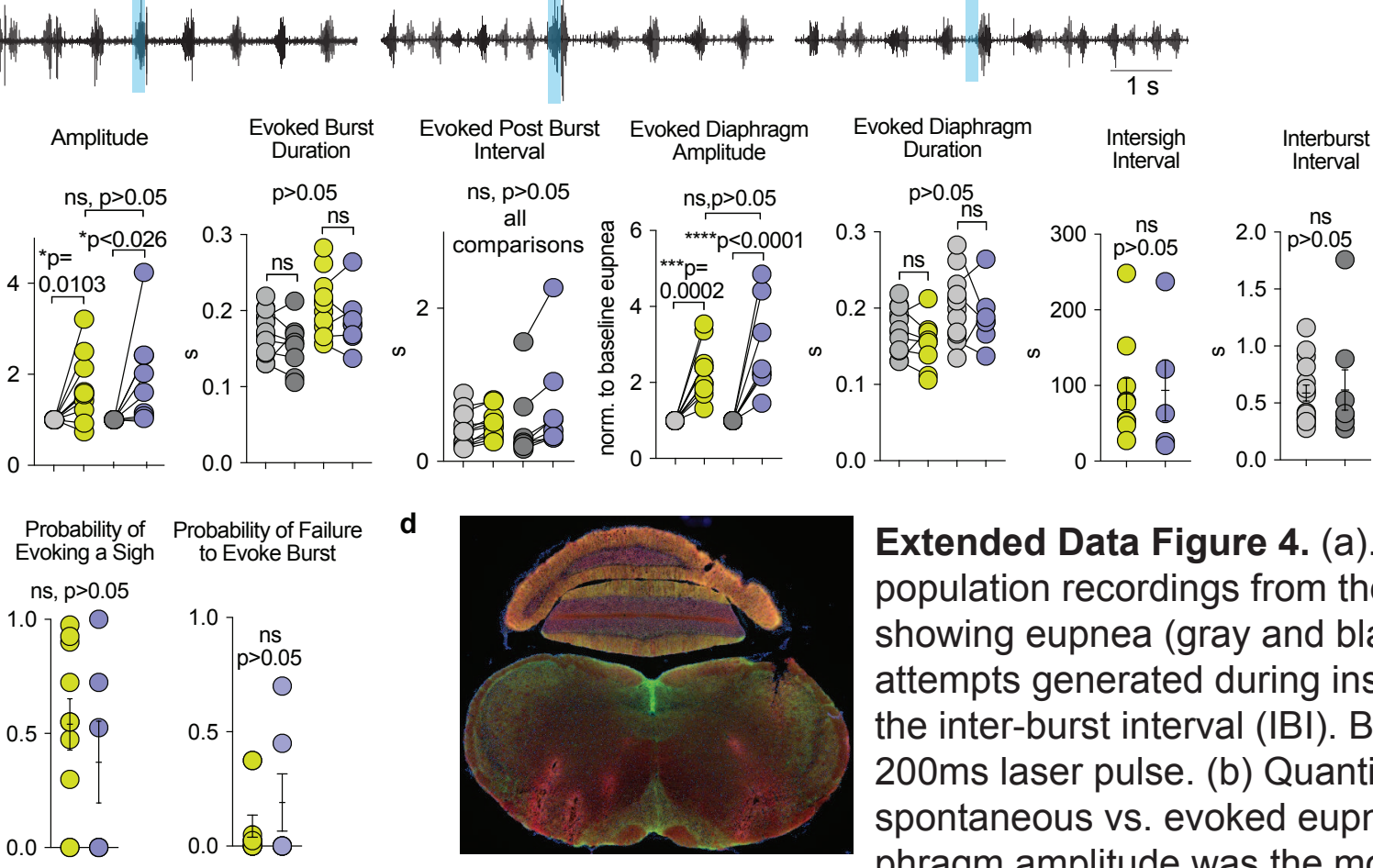

Extended Data Figure 4. (a). Representative population recordings from the preBötC in vitro showing eupnea (gray and black) and sighs/sigh attempts generated during inspiration and during the inter-burst interval $(\mathrm{IBI})$. Blue line represents $200 \mathrm{~ms}$ laser pulse. (b) Quantification of in vivo spontaneous vs. evoked eupnea and sighs. Diaphragm amplitude was the most defining

characteristic of sighs in vivo, as in XII nerve recordings evoked eupneic bursts had larger amplitudes than spontaneous eupneic bursts. (c) Evoked eupnea and sighs in vivo under baseline conditions and with application of MRS2279. MRS2279 was ineffective at blocking most sighs and limited the spontaneous and evoked response in a small number of experiments, regardless of injection location. (d) Representative MRS2279 injection sites located caudal to preBötC. 
Table 1: Neuron and Calcium Parameters

\begin{tabular}{|l|r|}
\hline \multicolumn{2}{|c|}{ Conductances } \\
\hline$C_{m}$ & $21 p F$ \\
$g_{N a}$ & $28 n S$ \\
$g_{K}$ & $11.2 n S$ \\
$g_{N a P}$ & $3.3 n S$ \\
$g_{\text {syn }}$ & $70 n S$ \\
$g_{\text {Cas }}$ & $0.5 n S$ \\
\hline
\end{tabular}

\begin{tabular}{|l|r|}
\hline \multicolumn{2}{|c|}{ Reversal Potentials } \\
\hline$E_{N a}$ & $50 m V$ \\
$E_{K}$ & $-85 m V$ \\
$E_{\text {leak }}$ & $-59 m V$ \\
$E_{C a s}$ & $0 m V$ \\
$E_{\text {syn }}$ & $0 m V$ \\
\hline
\end{tabular}

\begin{tabular}{|l|r|}
\hline \multicolumn{2}{|c|}{ Calcium Dynamics } \\
\hline$f_{i}$ & $0.001 \mathrm{pL}^{-1}$ \\
$L$ & $0.37 p L / s$ \\
$P$ & $31000 \mathrm{pL} / \mathrm{s}$ \\
$k_{I}$ & $1 \mu M$ \\
$k_{a}$ & $0.4 \mu M$ \\
$C_{t}$ & $1.25 \mu M$ \\
$\sigma$ & 0.185 \\
$V_{e}$ & $400 a M o l / s$ \\
$k_{e}$ & $0.2 \mu M$ \\
$A$ & $0.5 \mu M s^{-1}$ \\
$k_{d}$ & $0.4 \mu M$ \\
$\tau_{C a}$ & $1 s$ \\
\hline
\end{tabular}

Gating and kinetics

\begin{tabular}{|c|c|}
\hline$\overline{\tau_{n}}$ & $10 \mathrm{~ms}$ \\
\hline$\overline{\tau_{h}}$ & $10 \mathrm{~s}$ \\
\hline${\overline{\tau_{m}}}_{\text {Cas }}$ & $134 \mathrm{~ms}$ \\
\hline${\overline{\tau_{h}}}_{C a s}$ & $5.25 \mathrm{~s}$ \\
\hline$\tau_{0_{m_{C a s}}}$ & $2 m s$ \\
\hline 1. & $50 \mathrm{~ms}$ \\
\hline al & $500 n M$ \\
\hline
\end{tabular}

$[\mathrm{Ca}]_{h_{\text {Cas }}} \quad 510 n M$

\begin{tabular}{|l|r|}
$\xi_{m_{\text {Cas }}}$ & $-400 \mu M^{-1}$ \\
$\xi_{h_{\text {Cas }}}$ & $-250 \mu M^{-1}$ \\
$\theta_{m_{\text {Cas }}}$ & $-360 \mu M^{-1}$ \\
$\theta_{h_{\text {Cas }}}$ & $420 \mu M^{-1}$ \\
$V_{m}$ & $-34 m V$ \\
$V_{n}$ & $-29 m V$ \\
$V_{m_{p}}$ & $-40 m V$ \\
$V_{h}$ & $-48 m V$ \\
$\sigma_{m}$ & $-5 m V$ \\
$\sigma_{n}$ & $-4 m V$ \\
$\sigma_{m_{p}}$ & $-6 m V$ \\
$\sigma_{h}$ & $5 m V$ \\
$\tau_{s y n}$ & $15 m s$ \\
$Q_{s}$ & $15 m V$ \\
$\sigma_{s y n}$ & $-3 m V$ \\
\hline
\end{tabular}

Table 2: Network Parameters

$\mathrm{N}=300, K_{\text {avg }}=6$

\begin{tabular}{|l|c|r|}
\hline Neuron Type & $g_{\text {leak }}$ & \% of Pop. \\
\hline \hline Tonic & $U(0.5,1.5) n S$ & $10 \%$ \\
\hline Bursting & $U(2.5,3.5) n S$ & $60 \%$ \\
\hline Quiescent & $U(4.0,5.0) n S$ & $30 \%$ \\
\hline
\end{tabular}




\section{Figures}

a

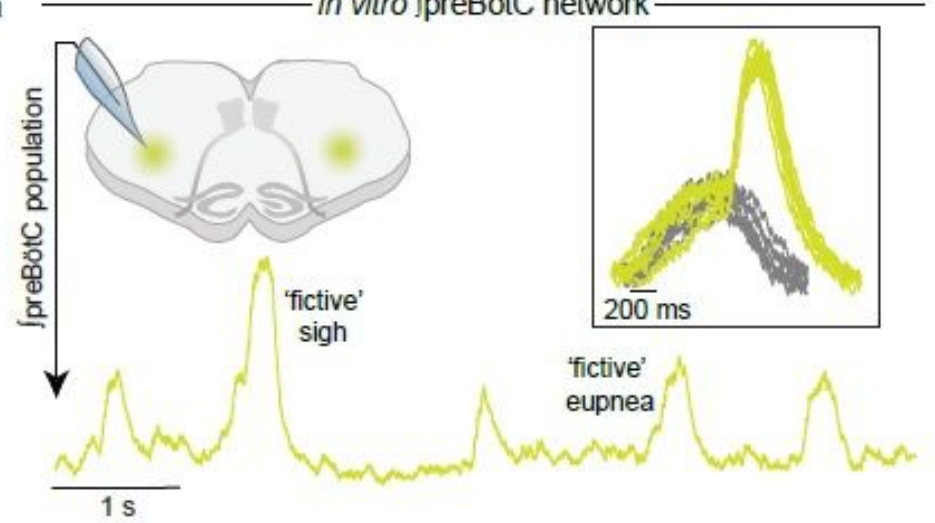

b

Amplitude Burst Duration $\begin{gathered}\begin{array}{c}\text { Post Burst } \\ \text { Interval }\end{array} \\ \begin{array}{c}\text { Inter-event } \\ \text { Interval }\end{array}\end{gathered}$

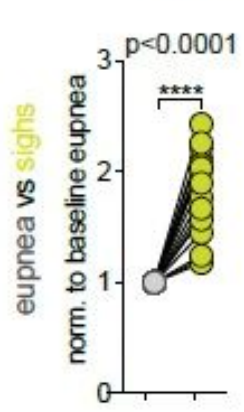

in vitro . jpreBötc network -

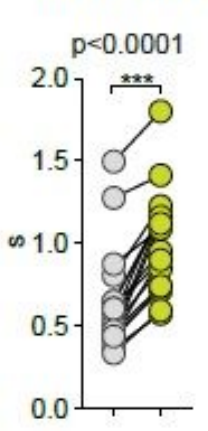

$\infty$

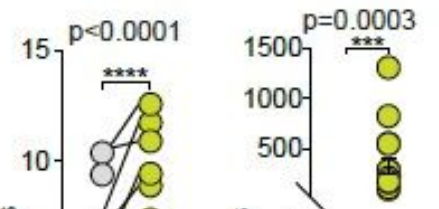

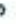

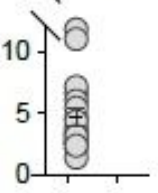

$$
\text { 。 }
$$

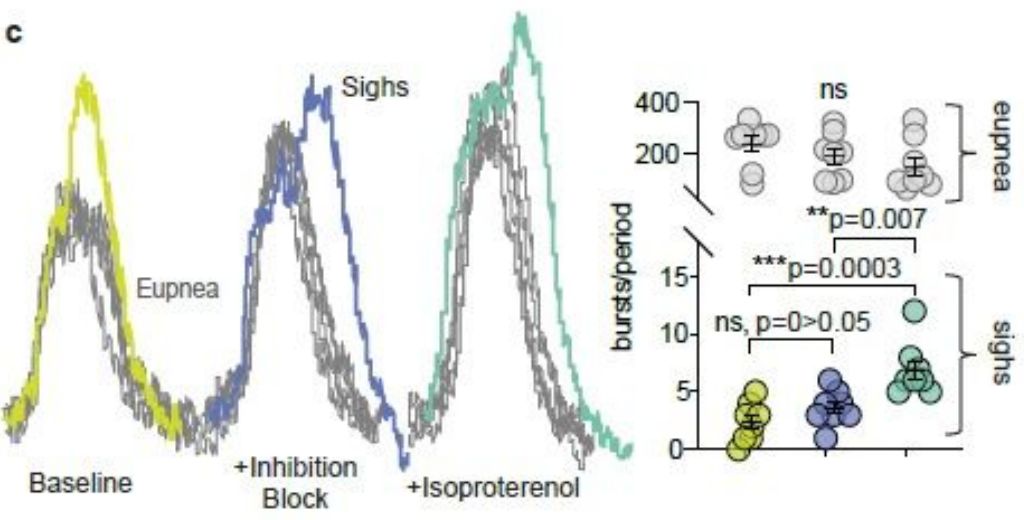

d
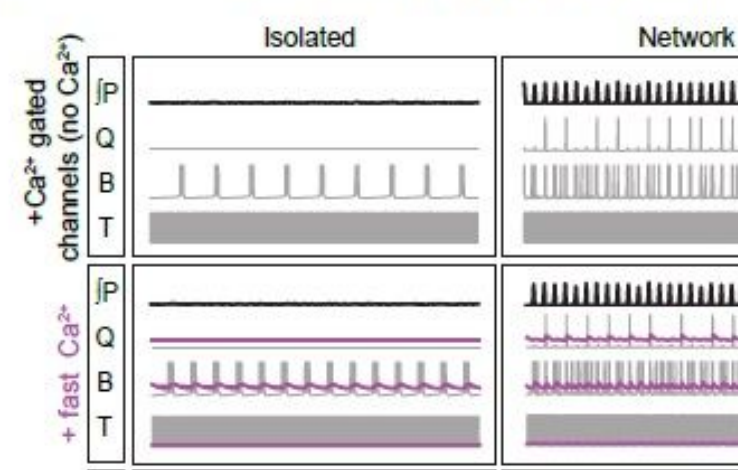

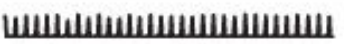

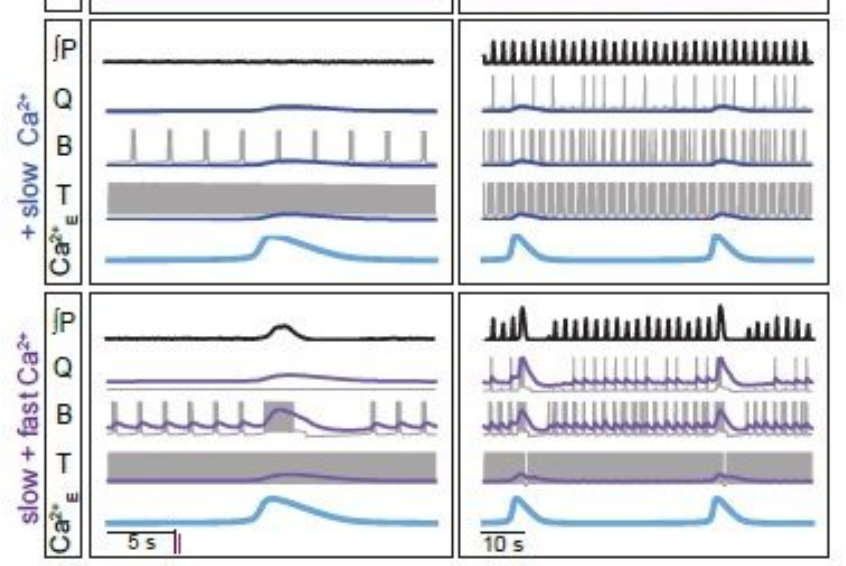

e



\section{Figure 1}

(see Manuscript file for figure legend) 
a

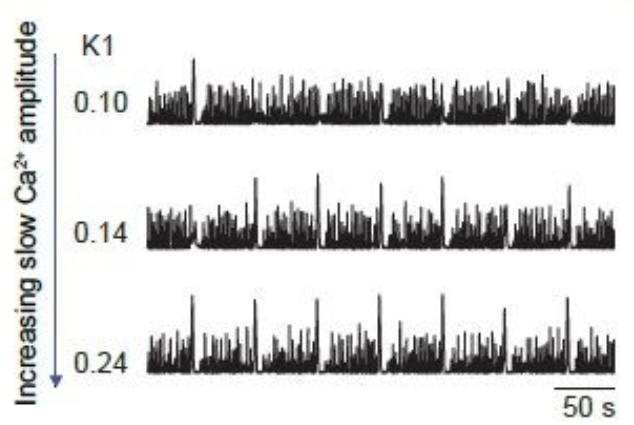

d

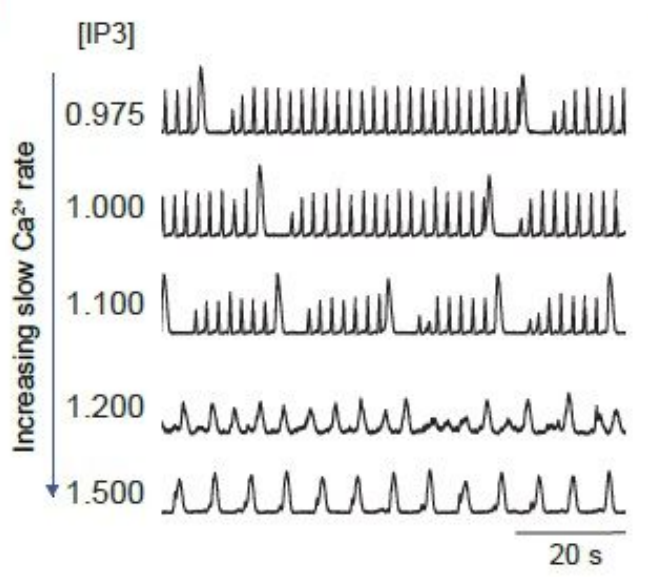

b

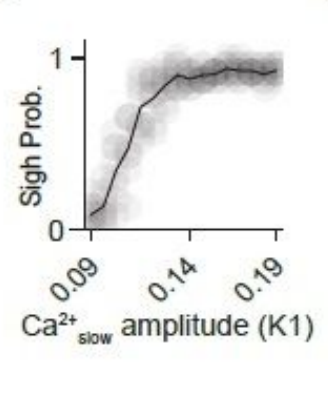

in vitro JpreBötC network

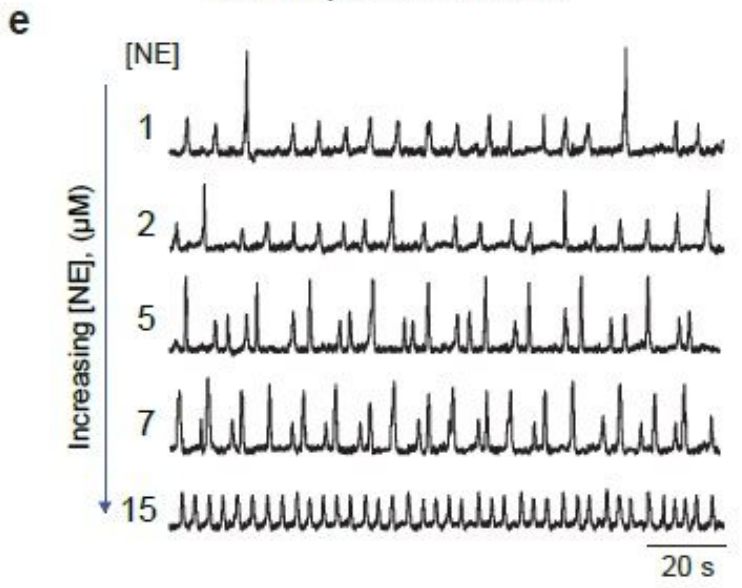

c

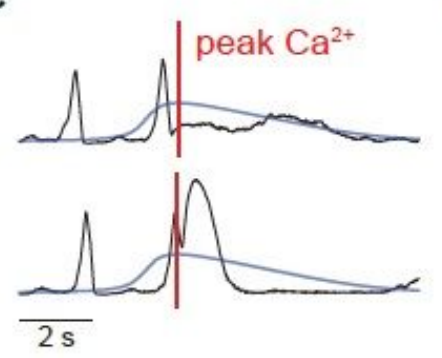

f $\mathrm{Ca}^{2+}{ }_{\text {slow }}$ amplitude (K1)

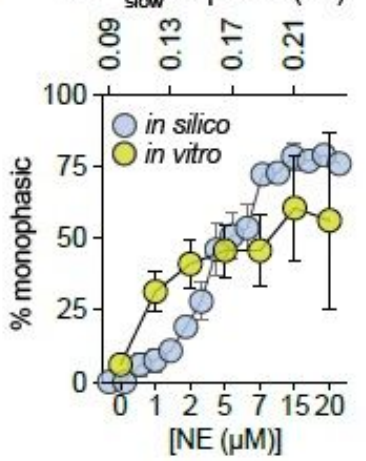

g

$\mathrm{Ca}_{\text {slow }}^{2+}$ frequency ([IP3])

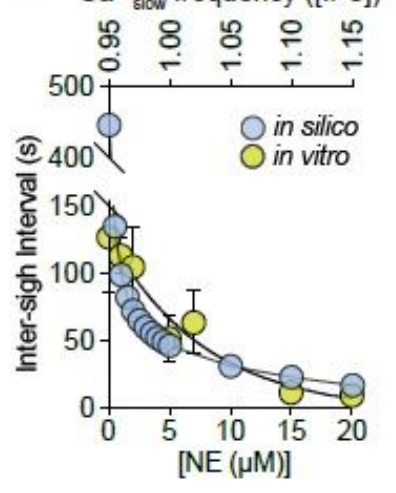

Figure 2

(see Manuscript file for figure legend) 
a
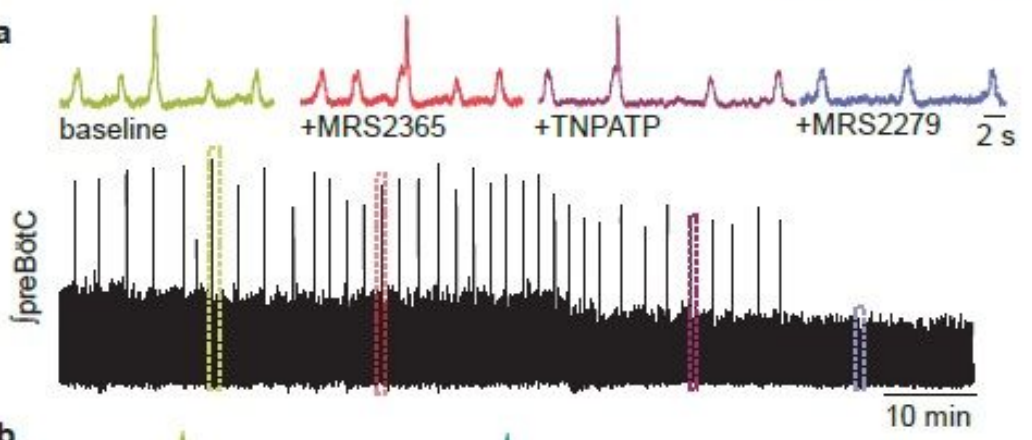

b
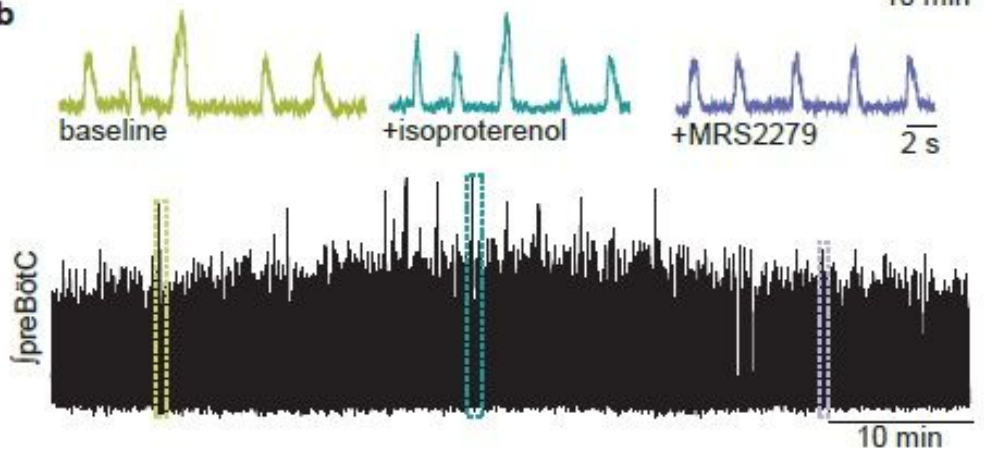

d severe hypoxia
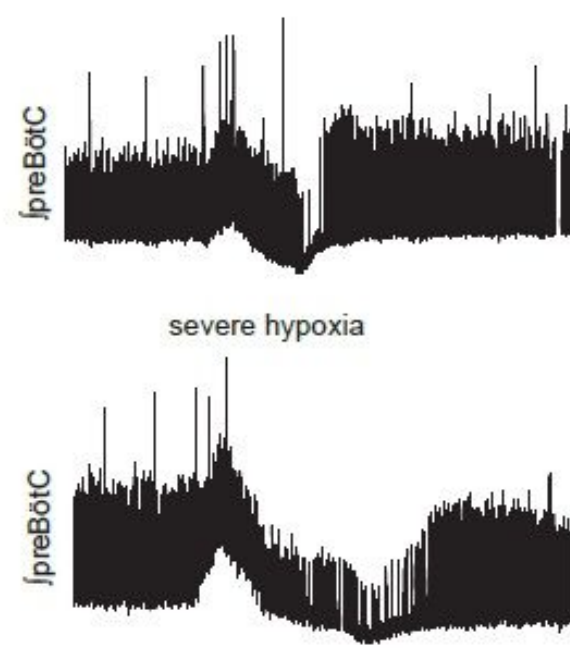

severe hypoxia
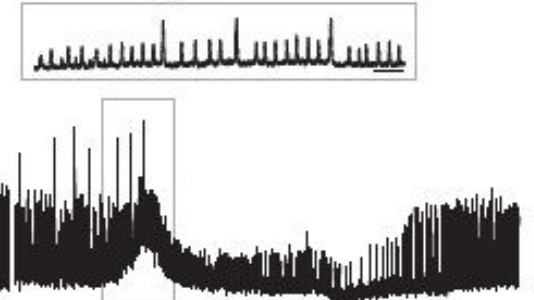

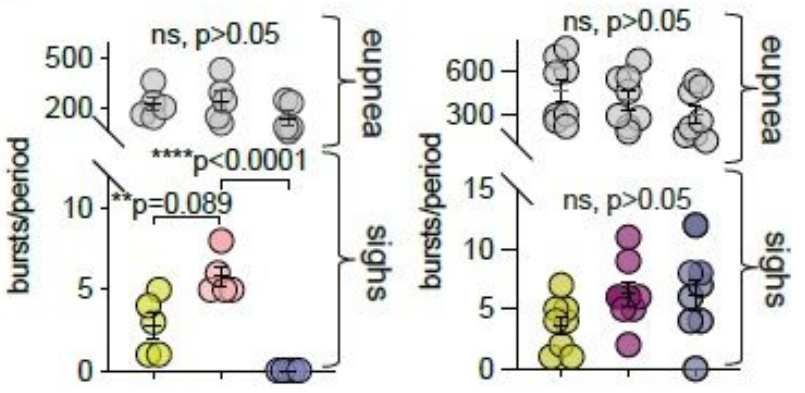

c
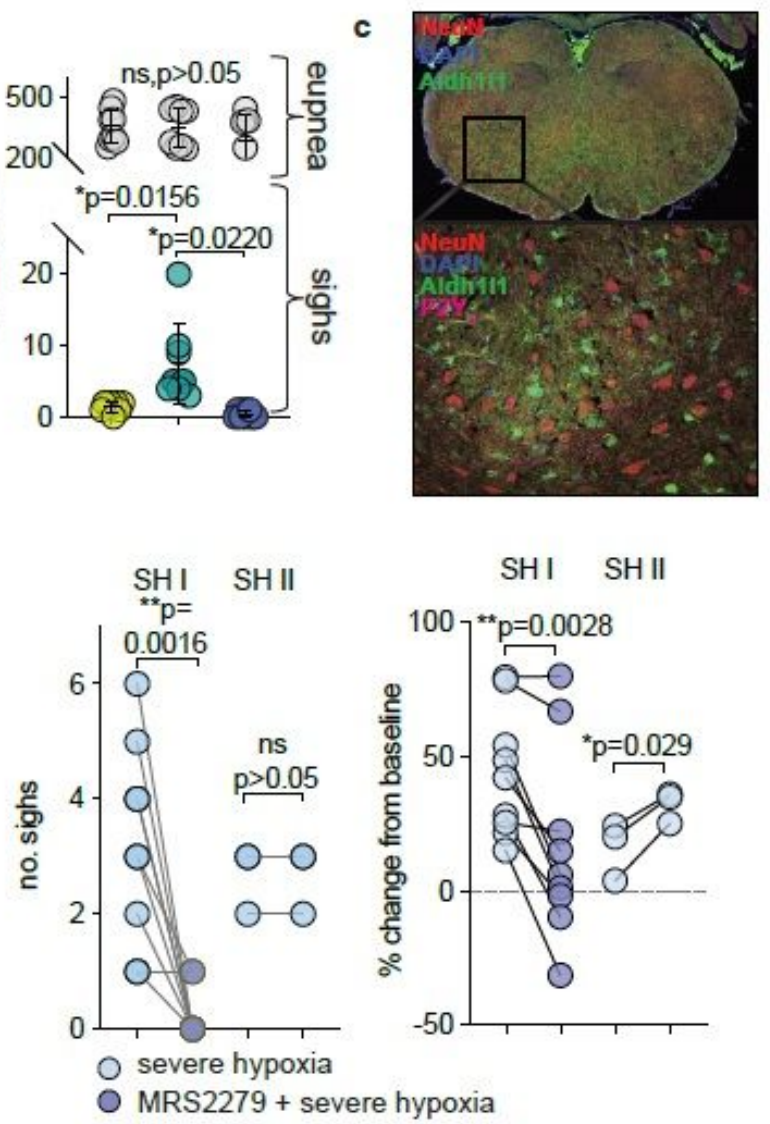

Figure 3

(see Manuscript file for figure legend) 

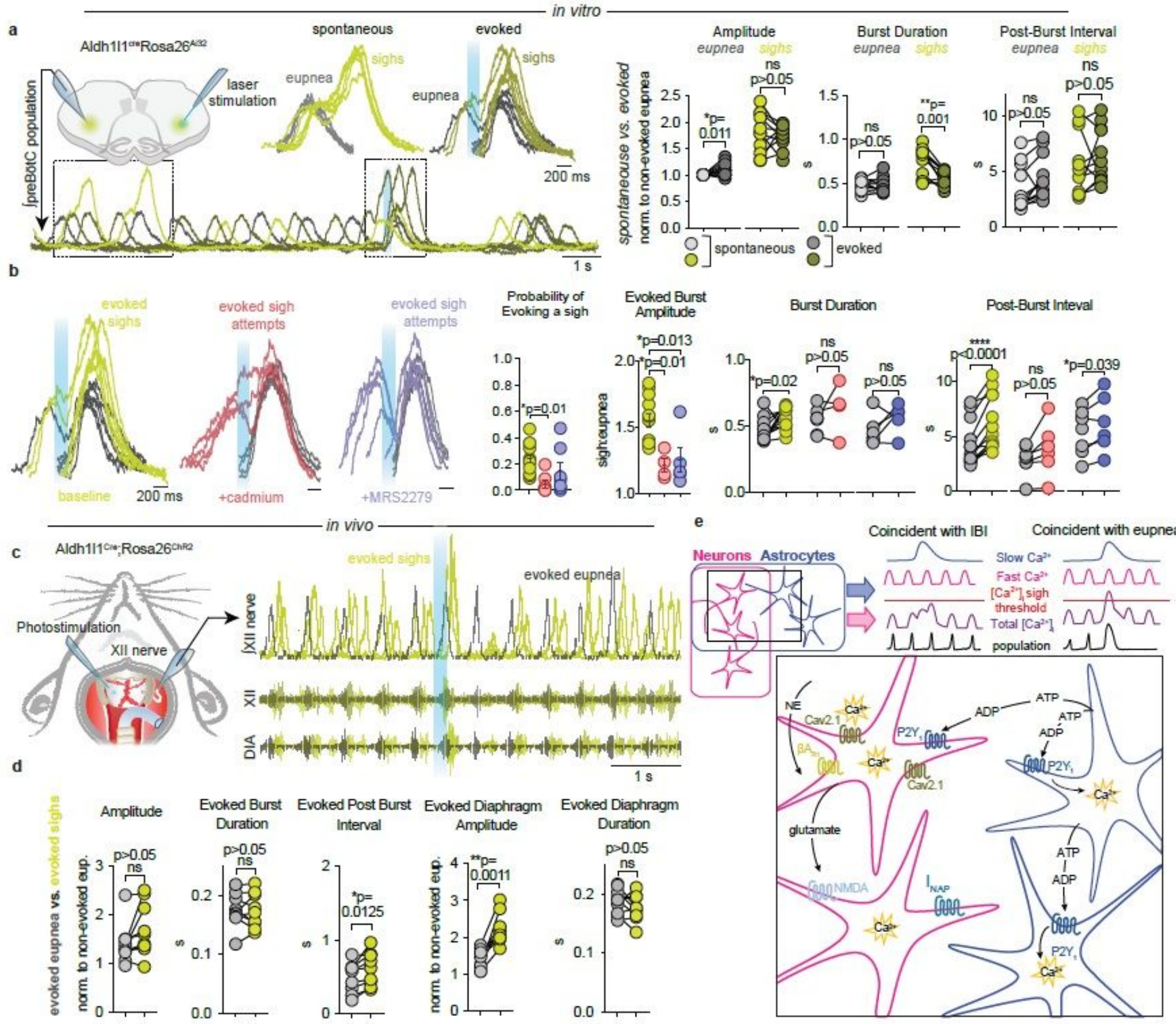

Figure 4

(see Manuscript file for figure legend) 